\title{
Local Analyticity in the Time and Space Variables and the Smoothing Effect for the Fifth-Order KdV-Type Equation
}

\author{
Kyoko Tomoeda \\ Graduate School of Humanities and Sciences, Nara Women's University, Nara 630-8506, Japan \\ Correspondence should be addressed to Kyoko Tomoeda, bak_tomoeda@yahoo.co.jp \\ Received 6 October 2010; Revised 23 December 2010; Accepted 26 January 2011 \\ Academic Editor: M. Lakshmanan
}

Copyright (C) 2011 Kyoko Tomoeda. This is an open access article distributed under the Creative Commons Attribution License, which permits unrestricted use, distribution, and reproduction in any medium, provided the original work is properly cited.

We consider the initial value problem for the reduced fifth-order KdV-type equation: $\partial_{t} u-\partial_{x}^{5} u-$ $10 \partial_{x}\left(u^{3}\right)+10 \partial_{x}\left(\partial_{x} u\right)^{2}=0, t, x \in \mathbb{R}, u(0, x)=\phi(x), x \in \mathbb{R}$. This equation is obtained by removing the nonlinear term $10 u \partial_{x}^{3} u$ from the fifth-order $\mathrm{KdV}$ equation. We show the existence of the local solution which is real analytic in both time and space variables if the initial data $\phi \in H^{s}(\mathbb{R})$ (s > $1 / 8)$ satisfies the condition $\sum_{k=0}^{\infty}\left(A_{0}^{k} / k !\right)\left\|\left(x \partial_{x}\right)^{k} \phi\right\|_{H^{s}}<\infty$, for some constant $A_{0} \quad\left(0<A_{0}<1\right)$. Moreover, the smoothing effect for this equation is obtained. The proof of our main result is based on the contraction principle and the bootstrap argument used in the third-order KdV equation (K. Kato and Ogawa 2000). The key of the proof is to obtain the estimate of $\partial_{x}\left(\partial_{x} u\right)^{2}$ on the Bourgain space, which is accomplished by improving Kenig et al.'s method used in (Kenig et al. 1996).

\section{Introduction}

The KdV hierarchy is well known as the series of the Lax pair formulation [1, 2], which are presented as

$$
\begin{aligned}
& \text { 1st-order } \mathrm{KdV} \partial_{t} u-\partial_{x} u=0 \text {, } \\
& \text { 3rd-order } \mathrm{KdV} \partial_{t} u+\partial_{x}^{3} u-6 u \partial_{x} u=0 \text {, } \\
& \text { 5th-order KdV } \partial_{t} u-\partial_{x}^{5} u-10 \partial_{x}\left(u^{3}\right)+10 \partial_{x}\left(\partial_{x} u\right)^{2}+10 u \partial_{x}^{3} u=0 \text {. }
\end{aligned}
$$


These equations describe mathematical models of some water waves [3,4]. We are interested in the existence theory of the analytic solution and the smoothing effect of the KdV hierarchy. There are some results concerning the analyticity for the third-order KdV equation (1.1) To. Kato and Masuda [5] considered the initial value problem of the following equation:

$$
\partial_{t} u+\partial_{x}^{3} u+a(u) \partial_{x} u=0, \quad t, x \in \mathbb{R}
$$

where $a(\lambda)$ is the real analytic and the no growth rate function in $\lambda \in \mathbb{R}$. They showed that if the initial data is real analytic, then, the global solution of (1.2) is real analytic in the space variable. Hayashi [6] also considered (1.2) in which $a(\lambda)$ is the polynomial. He showed that if the initial data is analytic and has an analytic continuation to a strip containing the real axis, then, the local solution also has the same property. When $a(u)=-6 u,(1.2)$ becomes the third-order KdV equation (1.1) ${ }_{1}$. K. Kato and Ogawa [7] proved that $(1.1)_{1}$ has not only the real analytic solution in both time and space variables but also the smoothing effect.

Recently, it is shown that the nonlinear dispersive equations including the $\mathrm{KdV}$ hierarchy have the local analytic solution in the space variable (see [8]). However, neither the existence of the real analytic solution in both time and space variables nor the smoothing effect is obtained for $(1.1)_{j}$ with $j \geq 2$, because the bilinear estimate of $u \partial_{x}^{2 j-1} u$ with $j \geq 2$ cannot be obtained by their method used in [9].

On the other hand, we may expect that the method used in [7] can work for the reduced equation given by removing $u \partial_{x}^{2 j-1} u$ from the higher-order $\mathrm{KdV}$ equations (1.1) with $j \geq 2$. In this paper, as a starting point for this attempt, we consider the following initial value problem of the reduced fifth-order $\mathrm{KdV}$-type equation:

$$
\begin{gathered}
\partial_{t} u-\partial_{x}^{5} u=\partial_{x}\left(u^{3}\right)+\partial_{x}\left(\partial_{x} u\right)^{2}, \quad t, x \in \mathbb{R}, \\
u(0, x)=\phi(x), \quad x \in \mathbb{R},
\end{gathered}
$$

where we may take all coefficients of the nonlinear terms to be equal to 1 without loss of generality. This equation is obtained by removing the nonlinear term $10 u \partial_{x}^{3} u$ from the original fifth-order KdV equation $(1.1)_{2}$. Our main purpose is to prove not only the existence of a local real analytic solution of (1.3) in both time and space variables but also the smoothing effect.

Before stating the main result precisely, we introduce the function space introduced by Bourgain (see [10]): for $s, b \in \mathbb{R}$, define that

$$
X_{b}^{s}=\left\{f \in \mathcal{S}^{\prime}\left(\mathbb{R}^{2}\right) ;\|f\|_{X_{b}^{s}}<\infty\right\},
$$

where

$$
\|f\|_{X_{b}^{s}}^{2}=\iint_{\mathbb{R}^{2}}\left(1+\left|\tau-\xi^{5}\right|\right)^{2 b}(1+|\xi|)^{2 s}\left|\Psi_{t, x} f(\tau, \xi)\right|^{2} d \tau d \xi
$$

and $\mathcal{F}_{t, x} f$ is the Fourier transform of $f$ in both $x$ and $t$ variables; that is,

$$
\mathcal{F}_{t, x} f(\tau, \xi)=(2 \pi)^{-1} \iint_{\mathbb{R}^{2}} f(t, x) e^{-i t \tau-i x \xi} d t d x
$$


Our main result is the following theorem.

Theorem 1.1. Let $s>1 / 8$ and let $b \in(1 / 2,23 / 40)$. Then, for any $\phi(x) \in H^{s}(\mathbb{R})$ such that

$$
\begin{gathered}
\left(x \partial_{x}\right)^{k} \phi(x) \in H^{s}(\mathbb{R}) \quad(k=0,1,2, \ldots), \\
\sum_{k=0}^{\infty} \frac{A_{0}^{k}}{k !}\left\|\left(x \partial_{x}\right)^{k} \phi\right\|_{H^{s}}<\infty, \quad \text { for some } 0<A_{0}<1,
\end{gathered}
$$

there exist a constant $T=T(\phi)>0$ and a unique solution $u \in C\left((-T, T), H^{s}\right) \cap X_{b}^{s}$ of (1.3) satisfying

$$
\begin{gathered}
P^{k} u \in C\left((-T, T), H^{s}\right) \cap X_{b}^{s}, \\
\sum_{k=0}^{\infty} \frac{A_{0}^{k}}{k !}\left\|P^{k} u\right\|_{X_{b}^{s}}<\infty
\end{gathered}
$$

where $P=5 t \partial_{t}+x \partial_{x}$ is the generator of dilation for the linear part of the equation of (1.3).

Moreover this solution becomes real analytic in both time and space variables; that is, there exist the positive constants $C$ and $A_{1}$ such that

$$
\left|\partial_{t}^{m} \partial_{x}^{l} u(t, x)\right| \leq C A_{1}^{m+l}(m+l) !
$$

holds for all $(t, x) \in(-T, 0) \cup(0, T) \times \mathbb{R}$ and $l, m=0,1,2, \ldots$

Remark 1.2. The initial data $\phi(x)$ has to be analytic except for $x=0$ but is allowed to have $H^{s}$-singularity at $x=0$. It follows from (1.9) that the singularity of $\phi(x)$ disappears after time passes and the regularity of the local solution of (1.3) reaches real analyticity in both time and space variables; that is, the fifth-order KdV-type equation has the smoothing effect.

Remark 1.3. A typical example of the initial data satisfying the condition (1.7) is given by

$$
|x|^{\gamma} e^{-x^{2}} \quad \text { with } \gamma>-\frac{3}{8}
$$

The existence results of the higher-order KdV equation are studied by many authors. Saut [11] and Schwarz [12] proved that each equation of the KdV hierarchy has a unique global solution in the spatially periodic Sobolev space. Kenig, Ponce, and Vega studied the initial value problem of the higher-order dispersive equation

$$
\partial_{t} u+\partial_{x}^{2 j+1} u+P\left(u, \partial_{x} u, \ldots, \partial_{x}^{2 j-1} u\right)=0
$$

where $j \geq 1$ and $P(\cdot)$ is a polynomial having no constant or linear part. They proved the local well-posedness in the weighted Sobolev space [13, 14]. Recently, Kwon [15] studied the simplified fifth-order KdV-type equation

$$
\partial_{t} u+\partial_{x}^{5} u+\partial_{x} u \partial_{x}^{2} u+u \partial_{x}^{3} u=0
$$


which is obtained by removing the term $10 \partial_{x}\left(u^{3}\right)$ from $(1.1)_{2}$. He showed the local wellposedness for the IVP of this equation in $H^{s}(\mathbb{R})$ with $s>5 / 2$. On the other hand, Ta. Kato [16] proved the following result for $(1.1)_{2}$.

Well-Posedness Theorem (Ta. Kato)

(1) Let

$$
s>-\frac{1}{4}, \quad s \geq-2 a-2, \quad \text { where }-\frac{3}{2}<a \leq-\frac{1}{4} .
$$

Then, the local well-posedness for the IVP of $(1.1)_{2}$ holds in $H^{s, a}(\mathbb{R})$, where

$$
H^{s, a}(\mathbb{R}) \equiv\left\{f \in \mathcal{S}^{\prime}(\mathbb{R}) ;\|f\|_{H^{s, a}} \equiv\left\|\langle\xi\rangle^{s-a}|\xi|^{a} \hat{f}\right\|_{L_{\xi}^{2}}<\infty\right\}
$$

(2) Let

$$
s \geq 1, \quad-\frac{1}{2} \leq a \leq-\frac{1}{4}
$$

Then, the global well-posedness for the IVP of $(1.1)_{2}$ holds in $H^{s, a}(\mathbb{R})$.

The plan of this paper is as follows. In Section 2, we give the existence and uniqueness of the local solution of (1.3), which is shown by the contraction argument consisting of Lemmas 2.3-2.5. In Section 3, we prove Lemma 2.4 which gives the bilinear estimate for $\partial_{x}\left(\partial_{x} u\right)^{2}$ in the Bourgain space. Kenig et al. [9] showed the bilinear estimate for $u \partial_{x} u$ of $(1.1)_{1}$ by estimating the potential which appears in an expression of the Bourgain norm of this term via duality. They divided the domain of integration of the potential into 17 subregions. However, their method of the domain decomposition is consistent with $(1.1)_{2}$, but not with the fifth-order KdV-type equation. We divide this domain into 30 subregions to derive the bilinear estimate for $\partial_{x}\left(\partial_{x} u\right)^{2}$. In Section 4 , we show the analyticity of the solution stated in Theorem 1.1 by the bootstrap argument. The result of this paper is announced in Proceedings of the Japan Academy [17].

Notation. Let $\mathcal{F}_{x}$ be the Fourier transform in the $x$ variable, and let $\mathcal{F}_{\xi}^{-1}$ and $\mathcal{F}_{\tau, \xi}^{-1}$ be the Fourier inverse transform in the $\xi$ and $(\tau, \xi)$ variables, respectively. The Riesz operator $D_{x}$ and its fractional derivative $\left\langle D_{x}\right\rangle^{s}$ are defined by

$$
D_{x}=\mathscr{F}_{\xi}^{-1}|\xi| \mathscr{F}_{x}, \quad\left\langle D_{x}\right\rangle^{s}=\mathcal{F}_{\xi}^{-1}\langle\xi\rangle^{s} \mathcal{F}_{x}
$$

respectively, where $\langle\cdot\rangle=(1+|\cdot|)$. Similarly, $\left\langle D_{t, x}\right\rangle^{S}$ is defined by

$$
\left\langle D_{t, x}\right\rangle^{s}=\mathscr{F}_{\tau, \xi}^{-1}\langle|\tau|+|\xi|\rangle^{s} \mathscr{F}_{t, x} .
$$


$[A, B]$ denotes the commutator relation of two operators given by $A B-B A . L_{t}^{p} L_{x}^{q}$ denotes the space $L^{p}\left(\mathbb{R}_{t} ; L^{q}\left(\mathbb{R}_{x}\right)\right)$ for $1 \leq p, q \leq \infty$ with the norm

$$
\|f\|_{L_{t}^{p} L_{x}^{q}}=\left(\int_{-\infty}^{\infty}\left(\int_{-\infty}^{\infty}|f(t, x)|^{p} d t\right)^{q / p} d x\right)^{1 / q} .
$$

We use Sobolev spaces with both time and space variables

$$
H_{t, x}^{s}\left(\mathbb{R}^{2}\right)=\left\{u \in \mathcal{S}^{\prime}\left(\mathbb{R}^{2}\right):\left\langle D_{t, x}\right\rangle^{s} u \in L_{t}^{2} L_{x}^{2}\right\},
$$

with the norm $\|\cdot\|_{H_{t, x}^{s}\left(\mathbb{R}^{2}\right)}=\left\|\left\langle D_{t, x}\right\rangle^{s} \cdot\right\|_{L_{t}^{2} L_{x}^{2}}$. Moreover, $L_{t}^{2}\left(\mathbb{R} ; H_{x}^{s}\right)$ denotes the space $L^{2}\left(\mathbb{R}_{t} ; H^{s}\left(\mathbb{R}_{x}\right)\right)$ with the norm $\|\cdot\|_{L_{t}^{2}\left(\mathbb{R} ; H_{x}^{s}\right)}=\left\|\left\langle D_{x}\right\rangle^{s} \cdot\right\|_{L_{t}^{2} L_{x}^{2}}$. The dual coupling is expressed as $\langle f, g\rangle$. The convolution of $f$ and $g$ with both space and time variables is denoted by $f * g$. For the constant $A_{0}$ appearing in Theorem 1.1, we put

$$
\mathscr{A}_{A_{0}}\left(X_{b}^{s}\right)=\left\{\mathbf{f}=\left(f_{0}, f_{1}, \ldots\right) ; f_{k} \in X_{b}^{s} \quad(k=0,1, \ldots),|\mathbf{f}|_{A_{A_{0}}\left(X_{b}^{s}\right)}<\infty\right\},
$$

where

$$
\|\mathbf{f}\|\left\|_{A_{A_{0}}\left(X_{b}^{s}\right)} \equiv \sum_{k=0}^{\infty} \frac{A_{0}^{k}}{k !}\right\| f_{k} \|_{X_{b}^{s}} .
$$

For simplicity we make use of three notations:

$$
\sum_{\mathbf{k}}=\sum_{k=k_{1}+k_{2}+k_{3}+k_{4}}, \quad \sum_{1}=\sum_{l=l_{1}+l_{2}+l_{3}}, \quad \sum_{\mathbf{m}}=\sum_{m=m_{1}+m_{2}+m_{3}} .
$$

\section{Existence and Uniqueness of the Solution}

In this section, we give the proof of the existence and uniqueness of the solution of (1.3). Let $u_{k}=P^{k} u$ and $\phi_{k}(x)=\left(x \partial_{x}\right)^{k} \phi(x)$, and we derive the equation which $u_{k}$ and $\phi_{k}(x)$ satisfy. Since $\left[x \partial_{x}, \partial_{x}\right]=-\partial_{x}$, it follows that

$$
(P+l)^{k} \partial_{x}=\partial_{x}(P+(l-1))^{k}, \quad k, l=0,1,2, \ldots
$$

Using (2.1) and the following relations

$$
\begin{gathered}
{\left[\partial_{t}-\partial_{x}^{5}, P\right]=5\left(\partial_{t}-\partial_{x}^{5}\right),} \\
\left(\partial_{t}-\partial_{x}^{5}\right) P^{k}=(P+5)^{k}\left(\partial_{t}-\partial_{x}^{5}\right),
\end{gathered}
$$


we have from (1.3)

$$
\begin{aligned}
& \partial_{t} u_{k}-\partial_{x}^{5} u_{k}=\mathcal{N}_{k}(u), \quad t, x \in \mathbb{R}, \quad k=0,1,2, \ldots, \\
& u_{k}(0, x)=\phi_{k}(x), \quad x \in \mathbb{R},
\end{aligned}
$$

where

$$
\mathcal{N}_{k}(u)=\partial_{x}(P+4)^{k}\left(u^{3}\right)+\partial_{x}(P+4)^{k}\left(\partial_{x} u\right)^{2}
$$

Using the Leibniz rule and (2.1), we can see that

$$
\begin{aligned}
\mathcal{N}_{k}(u) & =\partial_{x} \sum_{l=0}^{k}\left(\begin{array}{l}
k \\
l
\end{array}\right) 4^{k-l} P^{l}\left(u^{3}\right)+\partial_{x} \sum_{l=0}^{k}\left(\begin{array}{l}
k \\
l
\end{array}\right) 3^{k-l}(P+1)^{l}\left(\partial_{x} u\right)^{2} \\
& =\sum_{\mathbf{k}} \frac{k !}{k_{1} ! k_{2} ! k_{3} ! k_{4} !} 4^{k_{4}} \partial_{x}\left(u_{k_{1}} u_{k_{2}} u_{k_{3}}\right)+\sum_{\mathbf{k}} \frac{k !}{k_{1} ! k_{2} ! k_{3} ! k_{4} !} 3^{k_{4}}(-1)^{k_{3}} \partial_{x}\left(\left(\partial_{x} u_{k_{1}}\right)\left(\partial_{x} u_{k_{2}}\right)\right) .
\end{aligned}
$$

We will show the existence and uniqueness of the solution of (2.3).

Proposition 2.1. Let

$$
s>-\frac{1}{4}, \quad b \in\left(\frac{1}{2}, \frac{1}{2}+\sigma\right)
$$

where $\sigma=\min \{s / 5+1 / 20,3 / 16\}$. Then, for any $\phi \equiv\left(\phi_{0}, \phi_{1}, \ldots\right)$ such that $\phi_{k} \in H^{s}(\mathbb{R})(k=$ $0,1, \ldots)$ and

$$
|\|\phi\||_{A_{A_{0}}\left(H^{s}\right)}<\infty
$$

there exist a constant $T=T(\phi)>0$ and a unique solution $u_{k} \in C\left((-T, T), H^{s}\right) \cap X_{b}^{s}$ of (2.3) satisfying

$$
\|\mathbf{u}\|_{A_{A_{0}}\left(X_{b}^{s}\right)}<\infty, \quad \mathbf{u} \equiv\left(u_{0}, u_{1}, \ldots\right)
$$

Remark 2.2. The uniqueness of the solution of (2.3) yields $u_{k}=P^{k} u$ for $k=0,1,2, \ldots$ Moreover, $u_{0}$ becomes a solution of (1.3), the uniqueness of which also follows.

To prove this proposition we prepare three lemmas (Lemmas 2.3, 2.4 and 2.5), which play an important role in applying the contraction principle to the following system of the integral equations:

$$
\psi(t) u_{k}=\psi(t) e^{t \partial_{x}^{5}} \phi_{k}+\psi(t) \int_{0}^{t} e^{\left(t-t^{\prime}\right) \partial_{x}^{5}} \psi_{T}\left(t^{\prime}\right) \mathcal{N}_{k}(u)\left(t^{\prime}\right) d t^{\prime}
$$


where

$$
e^{t \partial_{x}^{5}} f \equiv \mathcal{F}_{\xi}^{-1}\left(e^{i \xi^{5} t} \widehat{f}(\xi)\right)
$$

$\psi(t)$ denotes a cut-off function in $C_{0}^{\infty}(\mathbb{R})$ satisfying

$$
\psi(t)= \begin{cases}1, & \text { if }|t| \leq 1 \\ 0, & \text { if }|t|>2\end{cases}
$$

and $\psi_{T}(t)=\psi(t / T)$.

Lemma 2.3. Let $0<T<1$ and let

$$
s \in \mathbb{R}, \quad b \in\left(\frac{1}{2}, 1\right), \quad a^{\prime}, a \in\left(0, \frac{1}{2}\right) \quad\left(a^{\prime}<a\right) .
$$

Then

$$
\begin{gathered}
\left\|\psi(t) e^{t \partial_{x}^{5}} \phi(x)\right\|_{X_{b}^{s}} \leq C_{0, s, b}\|\phi\|_{H^{s}} \\
\left\|\psi(t) \int_{0}^{t} e^{\left(t-t^{\prime}\right) \partial_{x}^{5}} h\left(t^{\prime}\right) d t^{\prime}\right\|_{X_{b}^{s}} \leq C_{1, s, b}\|h\|_{X_{b-1}^{s}}, \\
\left\|\psi_{T} h\right\|_{X_{-a}^{s}} \leq C_{2, s,-a,-a^{\prime}} T^{\left(a-a^{\prime}\right) / 4\left(1-a^{\prime}\right)}\|h\|_{X_{-a^{\prime}}^{s}},
\end{gathered}
$$

where $C_{0, s, b}, C_{1, s, b}$, and $C_{2, s,-a,-a^{\prime}}$ are constants depending on $s, b$, a and $a^{\prime}$.

Proof. Equations (2.13) and (2.14) are obtained by replacing the generator $e^{-t \partial_{x}^{3}}$ by $e^{t \partial_{x}^{5}}$ in the argument given by Kenig et al. [18] (see [19]). For the proof of (2.15), we refer to Lemma 2.5 in the study by Ginibre-Tsutsumi-Velo [20].

Lemma 2.4. Let

$$
s>-\frac{1}{4}, \quad b, b^{\prime} \in\left(\frac{1}{2}, \frac{1}{2}+\sigma\right) \quad\left(b \leq b^{\prime}\right),
$$

where $\sigma=\min \{s / 5+1 / 20,3 / 16\}$. Then

$$
\left\|\partial_{x}\left(\left(\partial_{x} u\right)\left(\partial_{x} v\right)\right)\right\|_{X_{b^{\prime}-1}^{s}} \leq C_{3, s, b, b^{\prime}}\|u\|_{X_{b}^{s}}\|v\|_{X_{b}^{s}}
$$

where $C_{3, s, b, b^{\prime}}$ is a constant depending on $s, b$, and $b^{\prime}$.

We give for the proof of this lemma, in Section 3. 
Lemma 2.5. Let

$$
s>-\frac{1}{4}, \quad b, b^{\prime} \in\left(\frac{1}{2}, \frac{3}{4}\right) \quad\left(b \leq b^{\prime}\right) .
$$

Then

$$
\left\|\partial_{x}(u v w)\right\|_{X_{b^{\prime}-1}^{s}} \leq C_{4, s, b, b^{\prime}}\|u\|_{X_{b}^{s}}\|v\|_{X_{b}^{s}}\|w\|_{X_{b}^{s}}
$$

where $C_{4, s, b, b^{\prime}}$ is a constant depending on $s, b$, and $b^{\prime}$.

Proof. This lemma is proved by improving Chen et al.'s argument used in the case where $b=b^{\prime} \in(1 / 2,3 / 4)[21]$.

Proof of Proposition 2.1. We define

$$
X_{M_{0}}=\left\{\mathbf{f} \in \mathcal{A}_{A_{0}}\left(X_{b}^{s}\right) ;|\|\mathbf{f}\||_{\mathcal{A}_{A_{0}}\left(X_{b}^{s}\right)} \leq 2 C_{0} M_{0}\right\}
$$

where

$$
C_{0}=C_{0, s, b,} \quad M_{0}=|\|\phi\||_{A_{A_{0}}\left(H^{s}\right)} .
$$

We define a map $\Phi: X_{M_{0}} \rightarrow X_{M_{0}}$ by $\Phi(u)=\left(\Phi_{0}(u), \Phi_{1}(u), \ldots\right)$ and

$$
\Phi_{k}(u)=\psi(t) e^{t \partial_{x}^{5}} \phi_{k}+\psi(t) \int_{0}^{t} e^{\left(t-t^{\prime}\right) \partial_{x}^{5}} \psi_{T}\left(t^{\prime}\right) \mathcal{N}_{k}(u)\left(t^{\prime}\right) d t^{\prime}
$$

Let $b^{\prime}$ and $T$ be positive constants satisfying $b<b^{\prime}<1 / 2+\sigma$ and

$$
T<\min \left\{1,\left(24 C_{0}^{2} C_{5} e^{4 A_{0}} M_{0}^{2}+8 C_{0} C_{6} e^{4 A_{0}} M_{0}\right)^{-1 / \mu}\right\}
$$

respectively, where

$$
C_{5}=C_{1, s, b} C_{2, s, b-1, b^{\prime}-1} C_{4, s, b, b^{\prime}} \quad C_{6}=C_{1, s, b} C_{2, s, b-1, b^{\prime}-1} C_{3, s, b, b^{\prime}} .
$$

We now show that $\Phi$ is a contraction mapping from $X_{M_{0}}$ to itself. According to Lemmas 2.3, 2.4, and 2.5, we have for $u \in \mathcal{A}_{A_{0}}\left(X_{b}^{s}\right)$

$$
\begin{aligned}
\left\|\Phi_{k}(u)\right\|_{X_{b}^{s}} \leq & C_{0}\left\|\Phi_{k}\right\|_{H^{s}}+C_{5} T^{\mu} \sum_{\mathbf{k}} \frac{k !}{k_{1} ! k_{2} ! k_{3} ! k_{4} !} 4^{k_{4}}\left\|u_{k_{1}}\right\|_{X_{b}^{s}}\left\|u_{k_{2}}\right\|_{X_{b}^{s}}\left\|u_{k_{3}}\right\|_{X_{b}^{s}} \\
& +C_{6} T^{\mu} \sum_{\mathbf{k}} \frac{k !}{k_{1} ! k_{2} ! k_{3} ! k_{4} !} 3^{k_{4}}\left\|u_{k_{1}}\right\|_{X_{b}^{s}}\left\|u_{k_{2}}\right\|_{X_{b}^{s}}
\end{aligned}
$$


for any $k \geq 0$. Here $\mu=\left(b^{\prime}-b\right) / 4 b^{\prime}>0$. By taking a sum over $k$, we have

$$
\begin{aligned}
\left.\|\Phi(u)\|\right|_{A_{A_{0}}\left(X_{b}^{s}\right)} \equiv & \sum_{k=0}^{\infty} \frac{A_{0}{ }^{k}}{k !}\left\|\Phi_{k}(u)\right\|_{X_{b}^{s}} \leq C_{0}|\|\phi\||_{A_{A_{0}}\left(H^{s}\right)} \\
& +C_{5} T^{\mu} \sum_{k_{4}=0}^{\infty} \frac{\left(4 A_{0}\right)^{k_{4}}}{k_{4} !} \sum_{k_{1}=0}^{\infty} \frac{A_{0}^{k_{1}}}{k_{1} !}\left\|u_{k_{1}}\right\|_{X_{b}^{s}} \sum_{k_{2}=0}^{\infty} \frac{A_{0}^{k_{2}}}{k_{2} !}\left\|u_{k_{2}}\right\|_{X_{b}^{s}} \sum_{k_{3}=0}^{\infty} \frac{A_{0}^{k_{3}}}{k_{3} !}\left\|u_{k_{3}}\right\|_{X_{b}^{s}} \\
& +C_{6} T^{\mu} \sum_{k_{4}=0}^{\infty} \frac{\left(3 A_{0}\right)^{k_{4}}}{k_{4} !} \sum_{k_{3}=0}^{\infty} \frac{A_{0}^{k_{3}}}{k_{3} !} \sum_{k_{1}=0}^{\infty} \frac{A_{0}^{k_{1}}}{k_{1} !}\left\|u_{k_{1}}\right\|_{X_{b}^{s}} \sum_{k_{2}=0}^{\infty} \frac{A_{0}^{k_{2}}}{k_{2} !}\left\|u_{k_{2}}\right\|_{X_{b}^{s}}
\end{aligned}
$$

Since $\mathbf{u} \in X_{M_{0}}$, we have from (2.23)

$$
\begin{aligned}
\left.\|\Phi(u)\|\right|_{A_{A_{0}}\left(X_{b}^{s}\right)} & \leq C_{0}|\|\phi\||_{A_{A_{0}}\left(H^{s}\right)}+C_{5} e^{4 A_{0}} T^{\mu}\|\mathbf{u}\|\left\|_{A_{A_{0}}\left(X_{b}^{s}\right)}^{3}+C_{6} e^{4 A_{0}} T^{\mu} \mid\right\| \mathbf{u}\|\|_{A_{A_{0}}\left(X_{b}^{s}\right)}^{2} \\
& \leq C_{0} M_{0}+8 C_{0}^{3} C_{5} e^{4 A_{0}} T^{\mu} M_{0}^{3}+4 C_{0}^{2} C_{6} e^{4 A_{0}} T^{\mu} M_{0}^{2} \\
& \leq \frac{3}{2} C_{0} M_{0}
\end{aligned}
$$

which implies $\Phi(u) \in X_{M_{0}}$. Similarly, we have for $u$ and $\tilde{u} \in \mathcal{A}_{A_{0}}\left(X_{b}^{s}\right)$

$$
\begin{aligned}
& |||\Phi(u)-\Phi(\tilde{u})|||_{A_{A_{0}}\left(X_{b}^{s}\right)} \\
& \leq C_{5} e^{4 A_{0}} T^{\mu} \\
& \times\left(|\|\mathbf{u}\||_{\mathcal{A}_{A_{0}}\left(X_{b}^{s}\right)}^{2}+|\|\mathbf{u}\||_{\mathcal{A}_{A_{0}}\left(X_{b}^{s}\right)}|\|\tilde{\mathbf{u}}\||_{\mathcal{A}_{A_{0}}\left(X_{b}^{s}\right)}+|\|\tilde{\mathbf{u}}\||_{\mathscr{A}_{A_{0}}\left(X_{b}^{s}\right)}^{2}\right)|\|\mathbf{u}-\tilde{\mathbf{u}}\||_{\mathcal{A}_{A_{0}}\left(X_{b}^{s}\right)} \\
& +C_{6} e^{4 A_{0}} T^{\mu}\left(|\|\mathbf{u}\||_{\mathcal{A}_{A_{0}}\left(X_{b}^{s}\right)}+|\|\tilde{\mathbf{u}}\||_{\mathcal{A}_{A_{0}}\left(X_{b}^{s}\right)}\right)|\|\mathbf{u}-\tilde{\mathbf{u}}\||_{\mathcal{A}_{A_{0}}\left(X_{b}^{s}\right)} \\
& \leq\left(12 C_{0}^{2} C_{5} e^{4 A_{0}} M_{0}^{2}+4 C_{0} C_{6} e^{4 A_{0}} M_{0}\right) T^{\mu}|\|\mathbf{u}-\tilde{\mathbf{u}}\||_{A_{A_{0}}\left(X_{b}^{s}\right)} \\
& \leq \frac{1}{2}|\|\mathbf{u}-\tilde{\mathbf{u}}\||_{A_{A_{0}}\left(X_{b}^{s}\right)} \text {. }
\end{aligned}
$$

Thus, the mapping $\Phi$ is contraction from $X_{M_{0}}$ to itself. We obtain a unique fixed point $u_{k} \in X_{b}^{s}$ satisfying

$$
u_{k}(t)=\psi(t) e^{-t \partial_{x}^{5}} \phi_{k}+\psi(t) \int_{0}^{t} e^{-\left(t-t^{\prime}\right) \partial_{x}^{5}} \psi_{T}\left(t^{\prime}\right) \mathcal{N}_{k}(u)\left(t^{\prime}\right) d t^{\prime}
$$

on the time interval $[-T, T]$ and $k=0,1,2, \ldots$ Uniqueness of the solution is also shown by using Bekiranov et al.'s argument in [22]. This completes the proof. 


\section{Proof of Lemma 2.4}

In this section we prove Lemma 2.4. To prove Lemma 2.4 we prepare the following lemma.

Lemma 3.1 (see [22]). (1) Let $\alpha, \beta>0$ and let $\kappa=\min \{\alpha, \beta\}$. If

$$
\alpha+\beta>1+\kappa,
$$

then

$$
\begin{array}{r}
\left(\int_{-\infty}^{\infty} \frac{d x}{(1+|x-\zeta|)^{\alpha}(1+|x-\eta|)^{\beta}}\right)^{1 / 2} \leq C_{7, \alpha, \beta}\left(\frac{1}{(1+|\zeta-\eta|)^{\kappa}}\right)^{1 / 2}, \\
\text { for any } \zeta, \eta \in \mathbb{R},
\end{array}
$$

where $C_{7, \alpha, \beta}$ is a constant depending on $\alpha$ and $\beta$.

(2) If $\gamma>1$, then

$$
\left(\int_{-\infty}^{\infty} \frac{d x}{(1+|x+\eta|)^{\gamma}}\right)^{1 / 2} \leq C_{8, \gamma}, \quad \text { for any } \eta \in \mathbb{R}
$$

where $C_{8, \gamma}$ is a constant depending on $\gamma$.

Proof of Lemma 2.4. By duality, we have

$$
\begin{aligned}
\left\|\partial_{x}\left(\left(\partial_{x} u\right)\left(\partial_{x} v\right)\right)\right\|_{X_{b^{\prime}-1}^{s}} & =\left\|\left\langle\tau-\xi^{5}\right\rangle^{\left(b^{\prime}-1\right)}\langle\xi\rangle^{s} \xi\left(\mathcal{F}_{t, x} \partial_{x} u\right) *\left(\mathcal{F}_{t, x} \partial_{x} u\right)\right\|_{L_{\tau}^{2} L_{\xi}^{2}} \\
& =\sup _{h \in L_{\tau}^{2} L_{\xi^{\prime}}^{2}\|h\|_{L_{\tau}^{2} L_{\xi}^{2}} \leq 1}\left|\left(\frac{\langle\xi\rangle^{s} \xi}{\left\langle\tau-\xi^{5}\right\rangle^{1-b^{\prime}}}\left(\mathcal{F}_{t, x} \partial_{x} u\right) *\left(\mathcal{F}_{t, x} \partial_{x} v\right), h\right)_{L_{\tau}^{2} L_{\xi}^{2}}\right|
\end{aligned}
$$

where $(\cdot, \cdot)_{L_{\tau}^{2} L_{\xi}^{2}}$ is the inner product in $L^{2}\left(\mathbb{R}_{\tau} \times \mathbb{R}_{\xi}\right)$. Setting

$$
f(\tau, \xi)=\left\langle\tau-\xi^{5}\right\rangle^{b}\langle\xi\rangle^{s} \mathcal{F}_{t, x} u(\tau, \xi), \quad g(\tau, \xi)=\left\langle\tau-\xi^{5}\right\rangle^{b}\langle\xi\rangle^{s} \mathcal{F}_{t, x} v(\tau, \xi),
$$


we have

$$
\begin{aligned}
& \left(\frac{\langle\xi\rangle^{s} \xi}{\left\langle\tau-\xi^{5}\right\rangle^{1-b^{\prime}}}\left(\mathcal{F}_{t, x} \partial_{x} u\right) *\left(\mathcal{F}_{t, x} \partial_{x} v\right), h\right)_{L_{\tau}^{2} L_{\xi}^{2}} \\
& =\iint_{\mathbb{R}^{2}} \frac{\langle\xi\rangle^{s} \xi}{\left\langle\tau-\xi^{5}\right\rangle^{1-b^{\prime}}} \\
& \quad \times\left(\iint_{\mathbb{R}^{2}} \frac{\xi_{1}\left(\xi-\xi_{1}\right) f\left(\tau_{1}, \xi_{1}\right) g\left(\tau-\tau_{1}, \xi-\xi_{1}\right)}{\left\langle\xi-\xi_{1}\right\rangle^{s}\left\langle\tau_{1}-\xi_{1}^{5}\right\rangle^{b}\left\langle\tau-\tau_{1}-\left(\xi-\xi_{1}\right)^{5}\right\rangle^{b}} d \tau_{1} d \xi_{1}\right) h(\tau, \xi) d \tau d \xi \\
& =I_{\Omega_{0.0 .0}}+I_{\Omega_{0.0 .0}{ }^{c},}
\end{aligned}
$$

where

$$
\begin{gathered}
I_{\Omega_{0.0 .0}}=\iiint \int_{\Omega_{0.0 .0}} \frac{\langle\xi\rangle^{s} \xi \xi_{1}\left(\xi-\xi_{1}\right) h(\tau, \xi) f\left(\tau_{1}, \xi_{1}\right) g\left(\tau-\tau_{1}, \xi-\xi_{1}\right)}{\left\langle\tau-\xi^{5}\right\rangle^{1-b^{\prime}}\left\langle\tau_{1}-\xi_{1}^{5}\right\rangle^{b}\left\langle\tau-\tau_{1}-\left(\xi-\xi_{1}\right)^{5}\right\rangle^{b}\left\langle\xi_{1}\right\rangle^{s}\left\langle\xi-\xi_{1}\right\rangle^{s}} d \tau_{1} d \xi_{1} d \tau d \xi \\
I_{\Omega_{0.0 .0} c}=\iiint \int_{\Omega_{0.0 .0}} \frac{\langle\xi\rangle^{s} \xi \xi_{1}\left(\xi-\xi_{1}\right) h(\tau, \xi) f\left(\tau_{1}, \xi_{1}\right) g\left(\tau-\tau_{1}, \xi-\xi_{1}\right)}{\left\langle\tau-\xi^{5}\right\rangle^{1-b^{\prime}}\left\langle\tau_{1}-\xi_{1}^{5}\right\rangle^{b}\left\langle\tau-\tau_{1}-\left(\xi-\xi_{1}\right)^{5}\right\rangle^{b}\left\langle\xi_{1}\right\rangle^{s}\left\langle\xi-\xi_{1}\right\rangle^{s}} d \tau_{1} d \xi_{1} d \tau d \xi, \\
\Omega_{0.0 .0}=\left\{\left(\tau, \tau_{1}, \xi, \xi_{1}\right) \in \mathbb{R}^{4}:|\xi|,\left|\xi_{1}\right|,\left|\xi-\xi_{1}\right| \leq 5\right\}, \quad \Omega_{0.0 .0}{ }^{c}=\mathbb{R}^{4} \backslash \Omega_{0.0 .0} .
\end{gathered}
$$

We split $\Omega_{0.0 .0}{ }^{c}$ into three regions, $\Omega_{1}, \Omega_{2}$, and $\Omega_{3}$ :

$$
\begin{aligned}
& \Omega_{1}=\left\{\left(\tau, \tau_{1}, \xi, \xi_{1}\right) \in \Omega_{0.0 .0}{ }^{c}:|\xi| \leq \frac{1}{4}\left|\xi_{1}\right|\right\}, \\
& \Omega_{2}=\left\{\left(\tau, \tau_{1}, \xi, \xi_{1}\right) \in \Omega_{0.0 .0}{ }^{c}: \frac{1}{4}\left|\xi_{1}\right| \leq|\xi| \leq 4\left|\xi_{1}\right|\right\}, \\
& \Omega_{3}=\left\{\left(\tau, \tau_{1}, \xi, \xi_{1}\right) \in \Omega_{0.0 .0}{ }^{c}: 4\left|\xi_{1}\right| \leq|\xi|\right\},
\end{aligned}
$$

and then, we split $\Omega_{i}(i=1,2,3)$ into three regions:

$$
\begin{aligned}
& \Omega_{i .1}=\left\{\left(\tau, \tau_{1}, \xi, \xi_{1}\right) \in \Omega_{i}:\left|\tau_{1}-\xi_{1}^{5}\right|,\left|\tau-\tau_{1}-\left(\xi-\xi_{1}\right)^{5}\right| \leq\left|\tau-\xi^{5}\right|\right\}, \\
& \Omega_{i .2}=\left\{\left(\tau, \tau_{1}, \xi, \xi_{1}\right) \in \Omega_{i}:\left|\tau-\xi^{5}\right|,\left|\tau-\tau_{1}-\left(\xi-\xi_{1}\right)^{5}\right| \leq\left|\tau_{1}-\xi_{1}^{5}\right|\right\}, \\
& \Omega_{i .3}=\left\{\left(\tau, \tau_{1}, \xi, \xi_{1}\right) \in \Omega_{i}:\left|\tau-\xi^{5}\right|,\left|\tau_{1}-\xi_{1}^{5}\right| \leq\left|\tau-\tau_{1}-\left(\xi-\xi_{1}\right)^{5}\right|\right\} .
\end{aligned}
$$


We further split $\Omega_{1 . j}, \Omega_{2 . j}$, and $\Omega_{3 . j}(j=1,2,3)$ into the following regions:

$$
\begin{aligned}
& \Omega_{1 . j .1}=\left\{\left(\tau, \tau_{1}, \xi, \xi_{1}\right) \in \Omega_{1 . j}:|\xi| \geq 1\right\}, \\
& \Omega_{1 . j .2}=\left\{\left(\tau, \tau_{1}, \xi, \xi_{1}\right) \in \Omega_{1 . j}:|\xi| \leq 1,|\xi|\left|\xi_{1}\right|^{4} \geq 1\right\}, \\
& \Omega_{1 . j .3}=\left\{\left(\tau, \tau_{1}, \xi, \xi_{1}\right) \in \Omega_{1 . j}:|\xi|\left|\xi_{1}\right|^{4} \leq 1\right\}, \\
& \Omega_{2.1 .1}=\left\{\left(\tau, \tau_{1}, \xi, \xi_{1}\right) \in \Omega_{2.1}:\left|\xi-\xi_{1}\right| \leq 1\right\}, \\
& \Omega_{2.1 .2}=\left\{\left(\tau, \tau_{1}, \xi, \xi_{1}\right) \in \Omega_{2.1}:\left|\xi-\xi_{1}\right| \geq 1,\left|\xi-2 \xi_{1}\right| \geq|\xi|^{-3 / 2}\right\}, \\
& \Omega_{2.1 .3}=\left\{\left(\tau, \tau_{1}, \xi, \xi_{1}\right) \in \Omega_{2.1}:\left|\xi-\xi_{1}\right| \geq 1,\left|\xi-2 \xi_{1}\right| \leq|\xi|^{-3 / 2}\right\}, \\
& \Omega_{2.2 .1}=\left\{\left(\tau, \tau_{1}, \xi, \xi_{1}\right) \in \Omega_{2.2}:\left|\xi-\xi_{1}\right| \leq 1\right\}, \\
& \Omega_{2.2 .2}=\left\{\left(\tau, \tau_{1}, \xi, \xi_{1}\right) \in \Omega_{2.2}:\left|\xi-\xi_{1}\right| \geq 1,\left|2 \xi-\xi_{1}\right| \geq\left|\xi_{1}\right|^{-3 / 2}\right\}, \\
& \Omega_{2.2 .3}=\left\{\left(\tau, \tau_{1}, \xi, \xi_{1}\right) \in \Omega_{2.2}:\left|\xi-\xi_{1}\right| \geq 1,\left|2 \xi-\xi_{1}\right| \leq\left|\xi_{1}\right|^{-3 / 2}\right\}, \\
& \Omega_{2.3 .1}=\left\{\left(\tau, \tau_{1}, \xi, \xi_{1}\right) \in \Omega_{2.3}:\left|\xi-\xi_{1}\right| \leq 1,\left|\xi-\xi_{1}\right|\left|\xi_{1}\right|^{4} \geq 1\right\}, \\
& \Omega_{2.3 .2}=\left\{\left(\tau, \tau_{1}, \xi, \xi_{1}\right) \in \Omega_{2.3}:\left|\xi-\xi_{1}\right|\left|\xi_{1}\right|^{4} \leq 1\right\}, \\
& \Omega_{2.3 .3}=\left\{\left(\tau, \tau_{1}, \xi, \xi_{1}\right) \in \Omega_{2.3}:\left|\xi-\xi_{1}\right| \geq 1,\left|\xi+\xi_{1}\right| \geq 1\right\}, \\
& \Omega_{2.3 .4}=\left\{\left(\tau, \tau_{1}, \xi, \xi_{1}\right) \in \Omega_{2.3}:\left|\xi-\xi_{1}\right| \geq 1,\left|\xi-\xi_{1}\right|^{-3 / 2} \leq\left|\xi+\xi_{1}\right| \leq 1\right\}, \\
& \Omega_{2.3 .5}=\left\{\left(\tau, \tau_{1}, \xi, \xi_{1}\right) \in \Omega_{2.3}:\left|\xi-\xi_{1}\right| \geq 1,\left|\xi+\xi_{1}\right| \leq\left|\xi-\xi_{1}\right|^{-3 / 2}\right\}, \\
& \Omega_{3 . j .1}=\left\{\left(\tau, \tau_{1}, \xi_{1} \xi_{1}\right) \in \Omega_{3 . j}:\left|\xi_{1}\right| \geq 1\right\}, \\
& \Omega_{3 . j .2}=\left\{\left(\tau, \tau_{1}, \xi_{,} \xi_{1}\right) \in \Omega_{3 . j}:\left|\xi_{1}\right| \leq 1,\left|\xi_{1}\right||\xi|^{4} \geq 1\right\}, \\
& \Omega_{3 . j .3}=\left\{\left(\tau, \tau_{1}, \xi, \xi_{1}\right) \in \Omega_{3 . j}:\left|\xi_{1}\right||\xi|^{4} \leq 1\right\},
\end{aligned}
$$

so that, we have

$$
\left|I_{\Omega_{0.0 .0}}+I_{\Omega_{0.0 .0}}\right| \leq\left|I_{\Omega_{0.0 .0}}\right|+\sum_{i, j, k=1}^{3}\left|I_{\Omega_{i, j, k}}\right|+\left|I_{\Omega_{2.3 .4}}\right|+\left|I_{\Omega_{2.3 .5}}\right| .
$$


Now we will estimate $\left|I_{\Omega_{0.0 .0}}\right|,\left|I_{\Omega_{i, j . k}}\right|(i, j, k=1,2,3)$, and $\left|I_{\Omega_{2.3 .1}}\right|(l=4,5)$. To estimate these terms, we prepare some estimates. By (3.8), (3.9) we obtain

$$
\begin{gathered}
\frac{3}{4}\left|\xi_{1}\right| \leq\left|\xi-\xi_{1}\right| \leq \frac{5}{4}\left|\xi_{1}\right|, \quad \text { in } \Omega_{1}, \\
\frac{3}{4}|\xi| \leq\left|\xi-\xi_{1}\right| \leq \frac{5}{4}|\xi|, \quad \text { in } \Omega_{3}, \\
\left|\xi_{1}\right| \geq 4, \quad \text { in } \Omega_{1} \cup \Omega_{2.2 .3}, \\
\min \left\{\left|\xi_{1}\right|,|\xi|\right\} \geq 1, \quad \text { in } \Omega_{2} \backslash\left\{\Omega_{4} \cup \Omega_{2.1 .3} \cup \Omega_{2.2 .3}\right\}, \\
\min \left\{\left|\xi_{1}\right|,|\xi|\right\} \geq 3, \quad \text { in } \Omega_{4}, \\
|\xi| \geq 4, \quad \text { in } \Omega_{3} \cup \Omega_{2.1 .3},
\end{gathered}
$$

where $\Omega_{4}=\Omega_{2.1 .1} \cup \Omega_{2.2 .1} \cup \Omega_{2.3 .1} \cup \Omega_{2.3 .2}$. Since (3.13) and (3.14) yield

$$
\left|\xi-\xi_{1}\right| \geq 3, \quad \text { in } \Omega_{1}, \Omega_{3},
$$

we have by (3.9) and (3.13)-(3.15)

$$
\begin{gathered}
\frac{\left|\xi_{1}\right|^{2}\left|\xi-\xi_{1}\right|^{2}}{\left\langle\xi_{1}\right\rangle^{2 s}\left\langle\xi-\xi_{1}\right\rangle^{2 s}} \leq C_{9, s}^{2}\left|\xi_{1}\right|^{4-4 s}, \quad \text { in } \Omega_{1}, \\
\frac{|\xi|^{2}\left|\xi_{1}\right|^{2}\langle\xi\rangle^{2 s}}{\left\langle\xi_{1}\right\rangle^{2 s}} \leq C_{9, s}^{2}\left|\xi_{1}\right|^{4}, \quad \text { in } \Omega_{2}, \\
\frac{|\xi|^{2}\left|\xi-\xi_{1}\right|^{2}\langle\xi\rangle^{2 s}}{\left\langle\xi-\xi_{1}\right\rangle^{2 s}} \leq C_{9, s}^{2}|\xi|^{4}, \quad \text { in } \Omega_{3},
\end{gathered}
$$

where $C_{9, s}=4^{|s|+1}$. Using (3.11), (3.14), and (3.15), we have

$$
\begin{gathered}
\langle\xi\rangle^{2 s} \leq 2^{2|s|} \max \{1,|\xi|\}^{2 s} \quad \text { in } \Omega_{1}, \\
\left\langle\xi-\xi_{1}\right\rangle^{-2 s} \leq 2^{2|s|} \max \left\{1,\left|\xi-\xi_{1}\right|\right\}^{-2 s} \text { in } \Omega_{2}, \\
\left\langle\xi_{1}\right\rangle^{-2 s} \leq 2^{2|s|} \max \left\{1,\left|\xi_{1}\right|\right\}^{-2 s} \text { in } \Omega_{3} .
\end{gathered}
$$


In $\Omega_{i_{1} \cdot j_{1} \cdot k_{1}}\left(\left(i_{1} \cdot j_{1} \cdot k_{1}\right)=(0.0 .0),(1.1 .3),(3.2 .1),(3.2 .2),(i .2 . k),(i=1,2, k=1,2,3)\right)$, we integrate with respect to $\tau$ and $\xi$ first, then, we use Schwarz's inequality, Fubini's theorem, and note that $\|h\|_{L_{\tau}^{2} L_{\xi}^{2}} \leq 1$ to have

$$
\begin{aligned}
& \left|I_{\Omega_{i_{1} \cdot j_{1}, k_{1}}}\right| \leq\|f\|_{L_{\tau_{1}}^{2} L_{\xi_{1}}^{2}} \\
& \times\left\|\frac{\left|\xi_{1}\right|}{\left\langle\tau_{1}-\xi_{1}^{5}\right\rangle^{b}\left\langle\xi_{1}\right\rangle^{s}}\left(\iint_{\mathbb{R}^{2}} \frac{h(\tau, \xi) g\left(\tau-\tau_{1}, \xi-\xi_{1}\right)\langle\xi\rangle^{s}|\xi|\left|\xi-\xi_{1}\right| \chi_{\Omega_{i_{1}, j_{1}, k_{1}}}\left(\tau, \tau_{1}, \xi, \xi_{1}\right) d \xi d \tau}{\left\langle\tau-\xi^{5}\right\rangle^{1-b^{\prime}}\left\langle\tau-\tau_{1}-\left(\xi-\xi_{1}\right)^{5}\right\rangle^{b}\left\langle\xi-\xi_{1}\right\rangle^{s}}\right)\right\|_{L_{\tau_{1}}^{2} L_{\xi_{1}}^{2}} \\
& \leq\|f\|_{L_{\tau}^{2} L_{\xi}^{2}} \|\left(\iint_{\mathbb{R}^{2}}|h(\tau, \xi)|^{2}\left|g\left(\tau-\tau_{1}, \xi-\xi_{1}\right)\right|^{2} d \xi d \tau\right)^{1 / 2}
\end{aligned}
$$

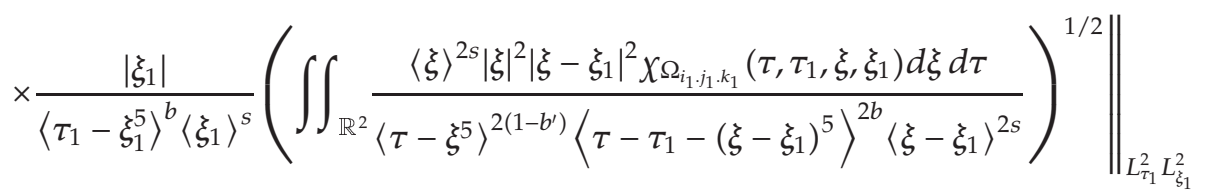

$$
\begin{aligned}
& \leq\|f\|_{L_{\tau}^{2} L_{\xi}^{2}}\|g\|_{L_{\tau}^{2} L_{\xi}^{2}} \\
& \times\left\|\frac{\left|\xi_{1}\right|}{\left\langle\tau_{1}-\xi_{1}^{5}\right\rangle^{b}\left\langle\xi_{1}\right\rangle^{s}}\left(\iint_{\mathbb{R}^{2}} \frac{\langle\xi\rangle^{2 s}|\xi|^{2}\left|\xi-\xi_{1}\right|^{2} \chi_{\Omega_{i_{1}, j_{1}, k_{1}}}\left(\tau, \tau_{1}, \xi, \xi \xi_{1}\right) d \xi d \tau}{\langle\tau\rangle^{2\left(1-b^{\prime}\right)}\left\langle\tau-\tau_{1}-\left(\xi-\xi_{1}\right)^{5}\right\rangle^{2 b}\left\langle\xi-\xi_{1}\right\rangle^{2 s}}\right)^{1 / 2}\right\|_{L_{\tau_{1}}^{\infty} L_{\xi_{1}}^{\infty}},
\end{aligned}
$$

where

$$
\chi \Omega_{i_{1} \cdot j_{1}, k_{1}}\left(\tau, \tau_{1}, \xi, \xi_{1}\right) \equiv \begin{cases}1, & \text { if }\left(\tau, \tau_{1}, \xi, \xi_{1}\right) \in \Omega_{i_{1} . j_{1} . k_{1}}, \\ 0, & \text { if }\left(\tau, \tau_{1}, \xi, \xi_{1}\right) \notin \Omega_{i_{1} . j_{1}, k_{1}} .\end{cases}
$$

In $\Omega_{i_{2} \cdot j_{2} \cdot k_{2}}\left(\left(i_{2} \cdot j_{2} \cdot k_{2}\right)=(i .1 . k),(i=1,2,3, k=1,2),(2.1 .3),(3.1 .3),(3.2 .3)\right)$, we integrate with respect to $\tau$ and $\xi$ first, then, we use the same way as in (3.18) to have

$$
\begin{aligned}
\left|I_{\Omega_{i_{2} \cdot j_{2}, k_{2}}}\right| \leq & \|f\|_{L_{\tau}^{2} L_{\xi}^{2}}\|g\|_{L_{\tau}^{2} L_{\xi}^{2}} \\
& \times\left\|\frac{\langle\xi\rangle^{s}|\xi|}{\left\langle\tau-\xi^{5}\right\rangle^{\left(1-b^{\prime}\right)}}\left(\iint_{\mathbb{R}^{2}} \frac{\left|\xi_{1}\right|^{2}\left|\xi-\xi_{1}\right|^{2} X \Omega_{i_{2}, j_{2}, k_{2}}\left(\tau, \tau_{1}, \xi, \xi_{1}\right) d \xi_{1} d \tau_{1}}{\left\langle\tau_{1}-\xi_{1}^{5}\right\rangle^{2 b}\left\langle\tau-\tau_{1}-\left(\xi-\xi_{1}\right)^{5}\right\rangle^{2 b}\left\langle\xi_{1}\right\rangle^{2 s}\left\langle\xi-\xi_{1}\right\rangle^{2 s}}\right)^{1 / 2}\right\|_{L_{\tau}^{\infty} L_{\xi}^{\infty}} .
\end{aligned}
$$


In $\Omega_{2.3 .2}$ we use the change of variables

$$
\tau_{2}=\tau_{1}-\tau, \quad \xi_{2}=\xi_{1}-\xi
$$

to obtain

$$
\begin{aligned}
& \iiint \int_{\Omega_{2.3 .2}} \frac{\langle\xi\rangle^{s} \xi \xi_{1}\left(\xi-\xi_{1}\right) h(\tau, \xi) f\left(\tau_{1}, \xi_{1}\right) g\left(\tau-\tau_{1}, \xi-\xi_{1}\right)}{\left\langle\tau \xi^{5}\right\rangle^{1-b^{\prime}}\left\langle\tau_{1}-\xi_{1}^{5}\right\rangle^{b}\left\langle\tau-\tau_{1}-\left(\xi-\xi_{1}\right)^{5}\right\rangle^{b}\left\langle\xi_{1}\right\rangle^{s}\left\langle\xi-\xi_{1}\right\rangle^{s}} d \tau_{1} d \xi_{1} d \tau d \xi \\
& =\iiint \int_{\widetilde{\Omega}_{2.3 .2}} \frac{\left\langle\xi_{1}-\xi_{2}\right\rangle^{s} \xi_{1}\left(\xi_{1}-\xi_{2}\right)\left(-\xi_{2}\right) h\left(\tau_{1}-\tau_{2}, \xi_{1}-\xi_{2}\right) f\left(\tau_{1}, \xi_{1}\right) g\left(-\tau_{2},-\xi_{2}\right)}{\left\langle\tau_{1}-\tau_{2}-\left(\xi-\xi_{1}\right)^{5}\right\rangle^{1-b^{\prime}}\left\langle\tau_{1}-\xi_{1}^{5}\right\rangle^{b}\left\langle\tau_{2}-\xi_{2}^{5}\right\rangle^{b}\left\langle\xi_{1}\right\rangle^{s}\left\langle\xi_{1}\right\rangle^{s}\left\langle\xi_{2}\right\rangle^{s}} d \tau_{1} d \xi_{1} d \tau_{2} d \xi_{2} \\
& \equiv J_{\widetilde{\Omega}_{2.3 .2}}
\end{aligned}
$$

where

$$
\begin{aligned}
\tilde{\Omega}_{2.3 .2}=\{ & \left(\tau_{1}, \tau_{2}, \xi_{1}, \xi_{2}\right) \in \Omega_{0.0 .0}{ }^{c}: \frac{1}{4}\left|\xi_{1}-\xi_{2}\right| \leq\left|\xi_{1}\right| \leq 4\left|\xi_{1}-\xi_{2}\right| \\
& \left.\left|\tau_{1}-\tau_{2}-\left(\xi_{1}-\xi_{2}\right)^{5}\right|,\left|\tau_{1}-\xi_{1}^{5}\right| \leq\left|\tau_{2}-\xi_{2}^{5}\right|,\left|\xi_{2}\right|\left|\xi_{1}\right|^{4} \leq 1\right\} .
\end{aligned}
$$

We integrate with respect to $\tau_{2}$ and $\xi_{2}$ first, then, we use the same way as in (3.18) to have

$$
\begin{aligned}
\left|J_{\tilde{\Omega}_{2.3 .2}}\right| & \leq\|f\|_{L_{\tau}^{2} L_{\xi}^{2}}\|g\|_{L_{\tau}^{2} L_{\xi}^{2}} \\
& \times\left\|\frac{\left|\xi_{1}\right|}{\left\langle\tau_{1}-\xi_{1}^{5}\right\rangle^{b}\left\langle\xi_{1}\right\rangle^{s}}\left(\iint_{\mathbb{R}^{2}} \frac{\left\langle\xi_{1}-\xi_{2}\right\rangle^{2 s}\left|\xi_{2}\right|^{2}\left|\xi_{1}-\xi_{2}\right|^{2} X_{\Omega_{2.3 .2}}\left(\tau_{1}, \tau_{2}, \xi_{1}, \xi_{2}\right) d \xi_{2} d \tau_{2}}{\left\langle\tau_{1}-\tau_{2}-\left(\xi_{1}-\xi_{2}\right)^{5}\right\rangle^{2\left(1-b^{\prime}\right)}\left\langle\tau_{2}-\xi_{2}^{5}\right\rangle^{2 b}\left\langle\xi_{2}\right\rangle^{2 s}}\right)^{1 / 2}\right\|_{L_{\tau_{1}}^{\infty} L_{\xi_{1}}^{\infty}} .
\end{aligned}
$$

In $\Omega_{i_{3} .3 . k_{3}}\left(\left(i_{3} .3 . k_{3}\right)=(i .3 . k),(i=1,3, k=1,2,3),(2.3 .1),(2.3 .3),(2.3 .4),(3.3 .5)\right)$, we have by a similar argument to (3.22)

$$
I_{\Omega_{i_{3}, 3 . k_{3}}}=J_{\tilde{\Omega}_{i_{3}, 3 . k_{3}}}
$$


where $\widetilde{\Omega}_{i_{3} .3 . k_{3}}$ is the region which is obtained from $\Omega_{i_{3} .3 . k_{3}}$ by the change of variables $\tau_{2}=\tau_{1}-\tau$ and $\xi_{2}=\xi_{1}-\xi$. We integrate with respect to $\tau_{1}$ and $\xi_{1}$ first, then, we use the same way as in (3.18) to have

$$
\begin{aligned}
& \left|J_{\tilde{\Omega}_{i_{3}, 3 . k_{3}}}\right| \leq\|f\|_{L_{\tau}^{2} L_{\xi}^{2}}\|g\|_{L_{\tau}^{2} L_{\xi}^{2}} \\
& \quad \times\left\|\frac{\left|\xi_{2}\right|}{\left\langle\tau_{2}-\xi_{2}^{5}\right\rangle^{b}\left\langle\xi_{2}\right\rangle^{s}}\left(\iint_{\mathbb{R}^{2}} \frac{\left\langle\xi_{1}-\xi_{2}\right\rangle^{2 s}\left|\xi_{1}\right|^{2}\left|\xi_{1}-\xi_{2}\right|^{2} X_{\tilde{\Omega}_{i_{3}, 3 . k_{3}}}\left(\tau_{1}, \tau_{2}, \xi_{1}, \xi_{2}\right) d \xi_{1} d \tau_{1}}{\left\langle\tau_{1}-\tau_{2}-\left(\xi_{1}-\xi_{2}\right)^{5}\right\rangle^{2\left(1-b^{\prime}\right)}\left\langle\tau_{1}-\xi_{1}^{5}\right\rangle^{2 b}\left\langle\xi_{1}\right\rangle^{2 s}}\right)^{1 / 2}\right\|_{L_{\tau_{2}}^{\infty} L_{\xi_{2}}^{\infty}} .
\end{aligned}
$$

Now we will get bounds for

$$
\begin{aligned}
& \left\|\frac{\left|\xi_{1}\right|}{\left\langle\tau_{1}-\xi_{1}^{5}\right\rangle^{b}\left\langle\xi_{1}\right\rangle^{s}}\left(\iint_{\mathbb{R}^{2}} \frac{\langle\xi\rangle^{2 s}|\xi|^{2}\left|\xi-\xi_{1}\right|^{2} \chi_{\Omega_{i_{1}, j_{1}, k_{1}}}\left(\tau, \tau_{1}, \xi, \xi_{1}\right) d \xi d \tau}{\langle\tau\rangle^{2\left(1-b^{\prime}\right)}\left\langle\tau-\tau_{1}-\left(\xi-\xi_{1}\right)^{5}\right\rangle^{2 b}\left\langle\xi-\xi_{1}\right\rangle^{2 s}}\right)^{1 / 2}\right\|_{L_{\tau_{1}}^{\infty} L_{\xi_{1}}^{\infty}}, \\
& \left\|\frac{\langle\xi\rangle^{s}|\xi|}{\left\langle\tau-\xi^{5}\right\rangle^{\left(1-b^{\prime}\right)}}\left(\iint_{\mathbb{R}^{2}} \frac{\left|\xi_{1}\right|^{2}\left|\xi-\xi_{1}\right|^{2} \chi_{\Omega_{i_{2}, j_{2}, k_{2}}}\left(\tau, \tau_{1}, \xi, \xi_{1}\right) d \xi_{1} d \tau_{1}}{\left\langle\tau_{1}^{5}\right\rangle^{2 b}\left\langle\tau-\tau_{1}-\left(\xi-\xi_{1}\right)^{5}\right\rangle^{2 b}\left\langle\xi_{1}\right\rangle^{2 s}\left\langle\xi-\xi_{1}\right\rangle^{2 s}}\right)^{1 / 2}\right\|_{L_{\tau}^{\infty} L_{\xi}^{\infty}}, \\
& \left\|\frac{\left|\xi_{1}\right|}{\left\langle\tau_{1}-\xi_{1}^{5}\right\rangle^{b}\left\langle\xi_{1}\right\rangle^{s}}\left(\iint_{\mathbb{R}^{2}} \frac{\left\langle\xi_{1}-\xi_{2}\right\rangle^{2 s}\left|\xi_{2}\right|^{2}\left|\xi_{1}-\xi_{2}\right|^{2} \chi_{\Omega_{2.3 .2}} d \xi_{2} d \tau_{2}}{\left\langle\tau_{1}-\tau_{2}-\left(\xi_{1}-\xi_{2}\right)^{5}\right\rangle^{2\left(1-b^{\prime}\right)}\left\langle\tau_{2}-\xi_{2}^{5}\right\rangle^{2 b}\left\langle\xi_{2}\right\rangle^{2 s}}\right)^{1 / 2}\right\|_{L_{\tau_{1}}^{\infty} L_{\xi_{1}}^{\infty}}, \\
& \left\|\frac{\left|\xi_{2}\right|}{\left\langle\tau_{2}-\xi_{2}^{5}\right\rangle^{b}\left\langle\xi_{2}\right\rangle^{s}}\left(\iint_{\mathbb{R}^{2}} \frac{\left\langle\xi_{1}-\xi_{2}\right\rangle^{2 s}\left|\xi_{2}\right|^{2}\left|\xi_{1}-\xi_{2}\right|^{2} X_{\Omega_{i_{3} .3 . k_{3}}} d \xi_{1} d \tau_{1}}{\left\langle\tau_{1}-\tau_{2}-\left(\xi_{1}-\xi_{2}\right)^{5}\right\rangle^{2\left(1-b^{\prime}\right)}\left\langle\tau_{1}-\xi_{1}^{5}\right\rangle^{2 b}\left\langle\xi_{1}\right\rangle^{2 s}}\right)^{1 / 2}\right\|_{L_{\tau_{2}}^{\infty} L_{\xi_{2}}^{\infty}} .
\end{aligned}
$$

By using the following methods, we estimate (3.27).

\section{The Case of $\Omega_{0.0 .0}$}

Since

$$
\left(\tau_{1}+\left(\xi-\xi_{1}\right)^{5}\right)-\xi^{5}=\tau_{1}-\xi_{1}^{5}-5 \xi \xi_{1}\left(\xi-\xi_{1}\right)\left(\xi^{2}-\xi \xi_{1}+\xi_{1}^{2}\right)
$$


it follows from (3.2) in Lemma 3.1 with $\alpha=2 b, \beta=\kappa=2\left(1-b^{\prime}\right)$ that

$$
\begin{aligned}
& \left\|\frac{\left|\xi_{1}\right|}{\left.\| \tau_{1}-\xi_{1}^{5}\right\rangle^{b}\left\langle\xi_{1}\right\rangle^{s}}\left(\iint_{\mathbb{R}^{2}} \frac{\langle\xi\rangle^{2 s}|\xi|^{2}\left|\xi-\xi_{1}\right|^{2} \chi \Omega_{0.0 .0}\left(\tau, \tau_{1}, \xi^{5}, \xi_{1}\right) d \xi d \tau}{\langle\tau\rangle^{2\left(1-b^{\prime}\right)}\left\langle\tau-\tau_{1}-\left(\xi-\xi_{1}\right)^{5}\right\rangle^{2 b}\left\langle\xi-\xi_{1}\right\rangle^{2 s}}\right)^{1 / 2}\right\|_{L_{\tau_{1}}^{\infty} L_{\xi_{1}}^{\infty}} \\
& \leq C_{7,2\left(1-b^{\prime}\right), 2 b} \\
& \quad \times\left\|\frac{\left|\xi_{1}\right| X_{\Omega_{B}}\left(\xi_{1}\right)}{\left\langle\tau_{1}-\xi_{1}^{5}\right\rangle^{b}\left\langle\xi_{1}\right\rangle^{s}}\left(\int_{\mathbb{R}} \frac{\langle\xi\rangle^{2 s}|\xi|^{2}\left|\xi-\xi_{1}\right|^{2} X \Omega_{A ; \xi_{1}}(\xi)}{\left\langle\tau_{1}-\xi_{1}^{5}-5 \xi_{1}\left(\xi-\xi_{1}\right)\left(\xi^{2}-\xi \xi_{1}+\xi_{1}^{2}\right)\right\rangle^{2\left(1-b^{\prime}\right)}\left\langle\xi-\xi_{1}\right\rangle^{2 s}} d \xi\right)^{1 / 2}\right\|_{L_{\tau_{1}}^{\infty} L_{\xi_{1}}^{\infty}},
\end{aligned}
$$

where $\Omega_{A ; \xi_{1}}=\left\{\xi:|\xi|,\left|\xi-\xi_{1}\right| \leq 5\right\}$ and $\Omega_{B}=\left\{\xi_{1}:\left|\xi_{1}\right| \leq 5\right\}$. Since $\langle\xi\rangle^{S} \leq \max \left\{1,6^{S}\right\}$, we have

$$
\begin{aligned}
& C_{7,2\left(1-b^{\prime}\right), 2 b} \\
& \quad \times\left\|\frac{\left|\xi_{1}\right| X_{\Omega_{B}}\left(\xi_{1}\right)}{\left\langle\tau_{1}-\xi_{1}^{5}\right\rangle^{b}\left\langle\xi_{1}\right\rangle^{s}}\left(\int_{\mathbb{R}} \frac{\langle\xi\rangle^{2 s}|\xi|^{2}\left|\xi-\xi_{1}\right|^{2} X \Omega_{A_{A ;} \xi_{1}}(\xi)}{\left\langle\tau_{1}-\xi_{1}^{5}-5 \xi \xi_{1}\left(\xi-\xi_{1}\right)\left(\xi^{2}-\xi_{1}+\xi_{1}^{2}\right)\right\rangle^{2\left(1-b^{\prime}\right)}\left\langle\xi-\xi_{1}\right\rangle^{2 s}} d \xi\right)^{1 / 2}\right\|_{L_{\tau_{1}}^{\infty} L_{\xi_{1}}^{\infty}} \\
& \leq C_{7,2\left(1-b^{\prime}\right), 2 b} \max \left\{1,6^{s}\right\} 5^{2}\left(\int_{|\xi| \leq 5}|\xi|^{2} d \xi\right)^{1 / 2} \\
& \leq M_{1, s, b, b^{\prime},}
\end{aligned}
$$

where $M_{1, s, b, b^{\prime}}$ is some constant.

The Case of $\Omega_{1 . j .3}, \Omega_{3 . j .3}(j=1,2), \tilde{\Omega}_{i .3 .3}(i=1,3)$ and $\tilde{\Omega}_{2.3 .2}$

We consider $\widetilde{\Omega}_{2.3 .2}$. By (3.2), we have

$$
\begin{aligned}
& \left\|\frac{\left|\xi_{1}\right|}{\|\left\langle\tau_{1}-\xi_{1}^{5}\right\rangle^{b}\left\langle\xi_{1}\right\rangle^{s}}\left(\iint_{\mathbb{R}} \frac{\left\langle\xi_{1}-\xi_{2}\right\rangle^{2 s}\left|\xi_{2}\right|^{2}\left|\xi-\xi_{1}\right|^{2} \chi_{\Omega_{2.3 .2}} d \xi_{2} d \tau_{2}}{\left\langle\tau_{1}-\tau_{2}-\left(\xi-\xi_{1}\right)^{5}\right\rangle^{2\left(1-b^{\prime}\right)}\left\langle\tau_{2}-\xi_{2}^{5}\right\rangle^{2 b}\left\langle\xi_{2}\right\rangle^{2 s}}\right)^{1 / 2}\right\|_{L_{\tau_{1}}^{\infty} L_{\xi_{1}}^{\infty}} \\
& \leq C_{7,2\left(1-b^{\prime}\right), 2 b} C_{9, s} 2^{|s|} \\
& \quad \times\left\|\frac{\left|\xi_{1}\right|^{2} X_{\Omega_{C}}\left(\xi_{1}\right)}{\left\langle\tau_{1}-\xi_{1}^{5}\right\rangle^{b}}\left(\int_{\mathbb{R}} \frac{\left|\xi_{2}\right|^{2} X_{\widetilde{\Omega}_{D ; \xi_{1}}}\left(\xi_{2}\right)}{\left\langle\tau_{1}-\xi_{1}^{5}+5 \xi_{1} \xi_{2}\left(\xi_{1}-\xi_{2}\right)\left(\xi_{1}^{2}-\xi_{1} \xi_{2}+\xi_{2}^{2}\right)\right\rangle^{2\left(1-b^{\prime}\right)}} d \xi_{2}\right)^{1 / 2}\right\|_{L_{\tau_{1}}^{\infty} L_{\xi_{1}}^{\infty}},
\end{aligned}
$$


where $\widetilde{\Omega}_{C}=\left\{\xi_{1}:\left|\xi_{1}\right| \geq 4\right\}$ and $\widetilde{\Omega}_{D ; \xi_{1}}=\left\{\xi_{2}:\left|\xi_{2}\right| \leq\left|\xi_{1}\right|^{-4}\right\}$. Here we have used (3.16) and (3.17) with the change of variables $\xi_{2}=\xi_{1}-\xi$. Noting

$$
2 b>0, \quad 2\left(1-b^{\prime}\right)>0,
$$

we have

$$
\begin{aligned}
& \left\|\frac{\left|\xi_{1}\right|^{2} \chi_{\tilde{\Omega}_{C}}\left(\xi_{1}\right)}{\left\langle\tau_{1}-\xi_{1}^{5}\right\rangle^{b}}\left(\int_{\mathbb{R}} \frac{\left|\xi_{2}\right|^{2} X_{\Omega_{\Omega_{D} ; \xi_{1}}}\left(\xi_{2}\right)}{\left\langle\tau_{1}-\xi_{1}^{5}+5 \xi_{1} \xi_{2}\left(\xi_{1}-\xi_{2}\right)\left(\xi_{1}^{2}-\xi_{1} \xi_{2}+\xi_{2}^{2}\right)\right\rangle^{2\left(1-b^{\prime}\right)}} d \xi_{2}\right)^{1 / 2}\right\|_{L_{\tau_{1}}^{\infty} \sum_{\xi_{1}}^{\infty}} \\
& \quad \leq\left\|\left\{\left|\xi_{1}\right|^{4} \chi_{\Omega_{C}}\left(\xi_{1}\right)\left(\int_{\left|\xi_{2}\right| \leq \xi_{1}||^{-4}}\left|\xi_{2}\right|^{2} d \xi_{2}\right)\right\}^{1 / 2}\right\|_{L_{\xi_{1}}^{\infty}} \\
& \quad \leq 2^{1 / 2}\left\|\left(\left|\xi_{1}\right|^{-8} X_{\Omega_{C}}\left(\xi_{1}\right)\right)^{1 / 2}\right\|_{L_{\xi_{1}}^{\infty}} \\
& \quad \leq 2^{1 / 2} .
\end{aligned}
$$

Thus, (3.27) is bounded by

$$
M_{2, s, b, b^{\prime}}=\left.C_{9, s}\right|^{|s|+1 / 2} \max \left\{C_{7,2\left(1-b^{\prime}\right), 2 b}, C_{7,2 b, 2 b}\right\}
$$

in $\widetilde{\Omega}_{2.3 .2}$. In the same manner as (3.31)-(3.33), (3.27) are bounded by $M_{2, s, b, b^{\prime}}$ in $\Omega_{1 . j .3}, \Omega_{3 . j .3}(j=$ $1,2)$, and $\widetilde{\Omega}_{i .3 .3}(i=1,3)$.

The Case of $\Omega_{2.1 .1}, \Omega_{2.2 .1}$

We consider $\Omega_{2.2 .1}$. Since

$$
\begin{aligned}
\left|\tau-\xi^{5}\right|+\left|\tau_{1}-\xi_{1}^{5}\right|+\left|\tau-\tau_{1}-\left(\xi-\xi_{1}\right)^{5}\right| \\
\quad \geq\left|\tau-\xi^{5}-\left(\tau_{1}-\xi_{1}^{5}\right)-\left(\tau-\tau_{1}-\left(\xi-\xi_{1}\right)^{5}\right)\right| \\
=5|\xi|\left|\xi_{1}\right|\left|\xi-\xi_{1}\right|\left|\xi^{2}-\xi \xi_{1}+\xi_{1}^{2}\right|
\end{aligned}
$$

we obtain

$$
\max \left\{\left|\tau-\xi^{5}\right|,\left|\tau_{1}-\xi_{1}^{5}\right|,\left|\tau-\tau_{1}-\left(\xi-\xi_{1}\right)^{5}\right|\right\} \geq \frac{5}{3}|\xi|\left|\xi_{1}\right|\left|\xi-\xi_{1}\right|\left|\xi^{2}-\xi \xi_{1}+\xi_{1}^{2}\right| .
$$

Noting that $-2 b<0$, we have

$$
\left\langle\tau_{1}-\xi_{1}^{5}\right\rangle^{-2 b} \leq 3^{2 b}\left\langle 5 \xi \xi_{1}\left(\xi-\xi_{1}\right)\left(\xi^{2}-\xi \xi_{1}+\xi_{1}^{2}\right)\right\rangle^{-2 b} \text { in } \Omega_{2.2 .1}
$$


Since (3.14) and $\left|\xi-\xi_{1}\right| \leq 1$ yield

$$
\left|\xi_{1}\right| \geq 3\left|\xi-\xi_{1}\right|
$$

we have

$$
\left|2 \xi-\xi_{1}\right| \geq\left|\xi_{1}\right|-2\left|\xi-\xi_{1}\right| \geq \frac{1}{3}\left|\xi_{1}\right|
$$

By (3.2), (3.16), and (3.37), we have

$$
\begin{aligned}
& \left\|\frac{\left|\xi_{1}\right|}{\|\left\langle\tau_{1}-\xi_{1}^{5}\right\rangle^{b}\left\langle\xi_{1}\right\rangle^{s}}\left(\iint_{\mathbb{R}^{2}} \frac{|\xi|^{2}\left|\xi-\xi_{1}\right|^{2}\langle\xi\rangle^{2 s} X \Omega_{22.1}\left(\tau, \tau_{1}, \xi^{5}\right\rangle^{2\left(1-b^{\prime}\right)}\left\langle\tau-\tau_{1}\right) d \tau d \xi}{\left\langle\xi-\left(\xi-\xi_{1}\right)^{5}\right\rangle^{2 b}\left\langle\xi-\xi_{1}\right\rangle^{2 s}}\right)^{1 / 2}\right\|_{L_{\tau_{1}}^{\infty} L_{\xi_{1}}^{\infty}} \\
& \leq C_{7,2\left(1-b^{\prime}\right), 2 b} C_{9, s} 2^{|s|} 3^{b} \\
& \quad \times\left\|\left(\int_{\mathbb{R}} \frac{\left\langle 5 \xi \xi_{1}\left(\xi-\xi_{1}\right)\left(\xi^{2}-\xi \xi_{1}+\xi_{1}^{2}\right)\right\rangle^{-2 b}\left|\xi_{1}\right|^{4}\left|\xi-\xi_{1}\right|^{2} X \Omega_{\Omega_{F ;} ;}\left(\xi_{1}\right)}{\left\langle\tau_{1}-\xi_{1}^{5}-5 \xi \xi_{1}\left(\xi-\xi_{1}\right)\left(\xi^{2}-\xi \xi_{1}+\xi_{1}^{2}\right)\right\rangle^{2\left(1-b^{\prime}\right)}} d \xi\right)^{1 / 2}\right\|_{L_{\tau_{1}}^{\infty} L_{\xi_{1}}^{\infty}},
\end{aligned}
$$

where $\Omega_{E ; \xi}=\left\{\xi_{1}:\left|\xi-\xi_{1}\right| \leq 1,\left|\xi_{1}\right| \geq 4\right\}$. Using the change of variable

$$
\mu=5 \xi \xi_{1}\left(\xi-\xi_{1}\right)\left(\xi^{2}-\xi \xi_{1}+\xi_{1}^{2}\right)
$$

and (3.2), we have

$$
\begin{aligned}
& \left\|\left(\int_{\mathbb{R}} \frac{\left\langle 5 \xi \xi_{1}\left(\xi-\xi_{1}\right)\left(\xi^{2}-\xi \xi_{1}+\xi_{1}^{2}\right)\right\rangle^{-2 b}\left|\xi_{1}\right|^{4}\left|\xi-\xi_{1}\right|^{2} \chi \Omega_{\Omega_{F} ;}\left(\xi_{1}\right)}{\left\langle\tau_{1}-\xi_{1}^{5}-5 \xi \xi_{1}\left(\xi-\xi_{1}\right)\left(\xi^{2}-\xi_{1} \xi_{1}+\xi_{1}^{2}\right)\right\rangle^{2\left(1-b^{\prime}\right)}} d \xi\right)^{1 / 2}\right\|_{L_{\tau_{1}}^{\infty} L_{\xi_{1}}^{\infty}} \\
& \quad \leq\left\|\left(\int_{|\mu| \leq 3\left|\tau_{1}-\xi_{1}^{5}\right|} \frac{\langle\mu\rangle^{-2 b}\left|\xi_{1}\right|^{4} d \mu}{\left\langle\tau_{1}-\xi_{1}^{5}-\mu\right\rangle^{2\left(1-b^{\prime}\right)} 5\left|\xi_{1}\right|\left|2 \xi-\xi_{1}\right|\left|\xi_{1}^{2}-2 \xi \xi_{1}+2 \xi^{2}\right|}\right)^{1 / 2}\right\|_{L_{\tau_{1}}^{\infty} L_{\xi_{1}}^{\infty}} \\
& \quad \leq\left\|\left(\int_{\mathbb{R}} \frac{(5 / 6)^{-1} d \mu}{\langle\mu\rangle^{2 b}\left\langle\mu-\left(\tau_{1}-\xi_{1}^{5}\right)\right\rangle^{2\left(1-b^{\prime}\right)}}\right)^{1 / 2}\right\|_{L_{\tau_{1}}^{\infty} L_{\xi_{1}}^{\infty}} \\
& \quad \leq C_{7,2\left(1-b^{\prime}\right), 2 b}\left(\frac{5}{6}\right)^{-1 / 2},
\end{aligned}
$$


where we have used

$$
5\left|\xi_{1}\right|\left|2 \xi-\xi_{1}\right|\left|\xi_{1}^{2}-2 \xi_{1}+2 \xi^{2}\right| \geq \frac{5}{6}\left|\xi_{1}\right|^{4} \quad \text { in } \Omega_{E ; \xi^{\prime}}
$$

which follows from (3.39) and

$$
\left|2 \xi^{2}-2 \xi \xi_{1}+\xi_{1}^{2}\right| \geq\left|2\left(\xi-\frac{1}{2} \xi_{1}\right)^{2}+\frac{1}{2} \xi_{1}^{2}\right| \geq \frac{1}{2}\left|\xi_{1}\right|^{2} .
$$

Hence (3.27) is bounded by

$$
M_{3, s, b, b^{\prime}}=\max \left\{C_{7,2\left(1-b^{\prime}\right), 2 b^{\prime}}^{2} C_{7,2\left(1-b^{\prime}\right), 2 b} C_{7,2 b, 2 b}\right\} C_{9, s} 2^{|s|}\left(3^{b}+3^{1-b^{\prime}}\right)\left(\frac{5}{6}\right)^{-1 / 2}
$$

in $\Omega_{2.2 .2}$. By a similar argument to (3.37)-(3.42), (3.27) is also bounded by $M_{3, s, b, b^{\prime}}$ in $\Omega_{2.2 .1}$.

The Case of $\Omega_{2.1 .3}, \Omega_{2.2 .3}$, and $\tilde{\Omega}_{2.3 .5}$

We consider $\Omega_{2.2 .3}$. Since (3.14) and $\left|2 \xi-\xi_{1}\right| \leq\left|\xi_{1}\right|^{-3 / 2}$ yield

$$
\left|2 \xi-\xi_{1}\right| \leq \frac{1}{2}\left|\xi_{1}\right|
$$

we have

$$
\begin{aligned}
\left|\xi-\xi_{1}\right| & \geq \frac{1}{2}\left(\left|\xi_{1}\right|-\left|2 \xi-\xi_{1}\right|\right) \geq \frac{1}{4}\left|\xi_{1}\right|, \\
\left|\xi-\xi_{1}\right| & \leq \frac{1}{2}\left(\left|\xi_{1}\right|+\left|2 \xi-\xi_{1}\right|\right) \leq \frac{3}{4}\left|\xi_{1}\right|,
\end{aligned}
$$

By (3.9) and (3.47), we have

$$
\frac{5}{3}|\xi|\left|\xi_{1} \| \xi-\xi_{1}\right|\left|\xi^{2}-\xi \xi_{1}+\xi_{1}^{2}\right| \geq \frac{5}{48}\left|\xi_{1}\right|^{5},
$$

where we have used

$$
\left|\xi^{2}-\xi \xi_{1}+\xi_{1}^{2}\right|=\left|\left(\xi-\frac{1}{2} \xi_{1}\right)^{2}+\frac{3}{4} \xi_{1}^{2}\right| \geq \frac{3}{4}\left|\xi_{1}\right|^{2} .
$$

Therefore using (3.36), we have

$$
\left\langle\tau_{1}-\xi_{1}^{5}\right\rangle^{-2 b} \leq\left(\frac{5}{48}\right)^{-2 b}\left|\xi_{1}\right|^{-5} \text { in } \Omega_{2.2 .3} .
$$


By (3.2), (3.16), (3.17), (3.47), and (3.50), we have

$$
\begin{aligned}
& \left\|\frac{\left|\xi_{1}\right|}{\left\langle\tau_{1}-\xi_{1}^{5}\right\rangle^{b}\left\langle\xi_{1}\right\rangle^{s}}\left(\iint_{\mathbb{R}^{2}} \frac{|\xi|^{2}\left|\xi-\xi_{1}\right|^{2}\langle\xi\rangle^{2 s} \chi_{\Omega_{2.2 .1}}\left(\tau, \tau_{1}, \xi, \xi_{1}\right) d \tau d \xi}{\left\langle^{2\left(1-b^{\prime}\right)}\left\langle\tau-\tau_{1}-\left(\xi-\xi_{1}\right)^{5}\right\rangle^{2 b}\left\langle\xi-\xi_{1}\right\rangle^{2 s}\right.}\right)^{1 / 2}\right\|_{L_{\tau_{1}}^{\infty} L_{\xi_{1}}^{\infty}} \\
& \leq C_{7,2\left(1-b^{\prime}\right), 2 b} C_{9, s} 2^{|s|} \max \left\{\left(\frac{1}{4}\right)^{1-s},\left(\frac{3}{4}\right)^{1-s}\right\}\left(\frac{5}{48}\right)^{-b} \\
& \times\left\|\left(\int_{\mathbb{R}} \frac{\left|\xi_{1}\right|^{-5}\left|\xi_{1}\right|^{4}\left|\xi_{1}\right|^{2-2 s} X \Omega_{G}\left(\xi_{1}\right) X \Omega_{\Omega_{F} ; 1}(\xi) d \xi}{\left\langle\tau_{1}-\xi_{1}^{5}-5 \xi \xi_{1}\left(\xi-\xi_{1}\right)\left(\xi^{2}-\xi \xi_{1}+\xi_{1}^{2}\right)\right\rangle^{2\left(1-b^{\prime}\right)}}\right)^{1 / 2}\right\|_{L_{\tau_{1}}^{\infty} \|_{\xi_{1}}^{\infty}},
\end{aligned}
$$

where $\Omega_{F ; \xi_{1}}=\left\{\xi:\left|2 \xi-\xi_{1}\right| \leq\left|\xi_{1}\right|^{-3 / 2}\right\}$ and $\Omega_{G}=\left\{\xi_{1}:\left|\xi_{1}\right| \geq 4\right\}$. Since $\left|2 \xi-\xi_{1}\right| \leq\left|\xi_{1}\right|^{-3 / 2}$, we have

$$
\begin{aligned}
& |\xi| \geq \frac{1}{2}\left(\left|\xi_{1}\right|-\left|2 \xi-\xi_{1}\right|\right) \geq \frac{1}{2}\left(\left|\xi_{1}\right|-\left|\xi_{1}\right|^{-3 / 2}\right), \\
& |\xi| \leq \frac{1}{2}\left(\left|\xi_{1}\right|-\left|2 \xi-\xi_{1}\right|\right) \leq \frac{1}{2}\left(\left|\xi_{1}\right|-\left|\xi_{1}\right|^{-3 / 2}\right),
\end{aligned}
$$

Noting

$$
s>-\frac{1}{4}, \quad 2\left(1-b^{\prime}\right)>0
$$

we have by (3.52)

$$
\begin{aligned}
& \left\|\left(\int_{\mathbb{R}} \frac{\left|\xi_{1}\right|^{-5}\left|\xi_{1}\right|^{4}\left|\xi_{1}\right|^{2-2 s} X \Omega_{\Omega_{G}}\left(\xi_{1}\right) \chi \Omega_{\Omega_{F ;}}(\xi) d \xi}{\left\langle\tau_{1}-\xi_{1}^{5}-5 \xi \xi_{1}\left(\xi-\xi_{1}\right)\left(\xi^{2}-\xi \xi_{1}+\xi_{1}^{2}\right)\right\rangle^{2\left(1-b^{\prime}\right)}}\right)^{1 / 2}\right\|_{L_{\tau_{1}}^{\infty} L_{\xi_{1}}^{\infty}} \\
& \quad \leq\left\|\left\{\left|\xi_{1}\right|^{1-2 s} X \Omega_{\Omega_{G}}\left(\xi_{1}\right)\left(\int_{\Omega_{H ; \xi_{1}}} 1 d \xi\right)\right\}^{1 / 2}\right\|_{L_{\tau_{1}}^{\infty} L_{\xi_{1}}^{\infty}} \\
& \quad \leq 1
\end{aligned}
$$

where

$$
\Omega_{H ; \xi_{1}}=\left\{\xi: \frac{1}{2}\left(\left|\xi_{1}\right|-\left|\xi_{1}\right|^{-3 / 2}\right) \leq|\xi| \leq \frac{1}{2}\left(\left|\xi_{1}\right|+\left|\xi_{1}\right|^{-3 / 2}\right)\right\}
$$


Therefore, (3.27) is bounded by

$$
M_{4, s, b, b^{\prime}}=C_{9, s} 2^{|s|} \max \left\{C_{7,2\left(1-b^{\prime}\right), 2 b}, C_{7,2 b, 2 b}\right\} \max \left\{\left(\frac{1}{4}\right)^{1-s},\left(\frac{3}{4}\right)^{1-s}\right\}
$$

in $\Omega_{2.2 .3}$. Using a similar argument to (3.47)-(3.54), we can get bounds of (3.27) in $\Omega_{2.1 .3}$ and $\tilde{\Omega}_{2.3 .5}$.

\section{All the Other Cases}

We consider $\Omega_{2.1 .2}$. By (3.9), we have

$$
\left|\xi-\xi_{1}\right| \leq|\xi|+\left|\xi_{1}\right| \leq 5|\xi|
$$

Since $2 b^{\prime}<1+2 \sigma$ and $\sigma=\min \{s / 5+1 / 20,3 / 16\}$ yield

$$
-\frac{3}{2}+4\left(2 b^{\prime}-1\right)<-\frac{3}{2}+8 \sigma<0,
$$

we have by (3.16), (3.36), and (3.57)

$$
\begin{gathered}
\frac{|\xi|^{2}\left|\xi_{1}\right|^{2}\langle\xi\rangle^{2 s}}{\left\langle\xi_{1}\right\rangle^{2 s}} \leq C_{9, s}^{2} 5^{3 / 2}|\xi|^{11 / 2-8 \sigma}\left|\xi-\xi_{1}\right|^{-3 / 2+8 \sigma}, \\
\left\langle\tau-\xi^{5}\right\rangle^{2\left(b^{\prime}-1\right)} \leq\left(\frac{5}{16}\right)^{2\left(b^{\prime}-1\right)}\left(\left|\xi-\xi_{1} \| \xi\right|^{4}\right)^{-1+2 \sigma},
\end{gathered}
$$

By (3.2), (3.17), and (3.59), we have

$$
\begin{gathered}
\left\|\frac{\langle\xi\rangle^{2 s}|\xi|^{2}}{\left\langle\tau-\xi^{5}\right\rangle^{1-b^{\prime}}}\left(\iint_{\mathbb{R}^{2}} \frac{\left.\left|\xi_{1}\right|^{2}\left|\xi-\xi_{1}\right|^{2} \chi_{\Omega_{2.12}}\left(\tau, \tau_{1}, \xi_{1}, \xi_{1}\right) d \xi_{1}^{5}\right\rangle^{2 b}\left\langle\tau-\tau_{1}-\left(\xi-\xi_{1}\right)^{5}\right\rangle^{2 b}\left\langle\xi_{1}\right\rangle^{2 s}\left\langle\xi-\xi_{1}\right\rangle^{2 s}}{{ }^{2 s}}\right)^{1 / 2}\right\|_{L_{\tau}^{\infty} L_{\xi}^{\infty}} \\
\leq C_{7,2 b, 2 b} C_{9, s} 2^{|s|} 5^{3 / 4}\left(\frac{5}{16}\right)^{\left(b^{\prime}-1\right)} \\
\quad \times\left\|\left(\int_{\mathbb{R}} \frac{|\xi|^{(11 / 2)-8 \sigma}\left|\xi-\xi_{1}\right|^{(1 / 2)-2 s+8 \sigma} \chi_{\Omega_{I, \xi}}\left(\xi_{1}\right) d \xi_{1}}{\left(\left|\xi-\xi_{1}\right||\xi|^{4}\right)^{1-2 \sigma}\left\langle\tau-\xi^{5}+5 \xi \xi_{1}\left(\xi-\xi_{1}\right)\left(\xi^{2}-\xi \xi_{1}+\xi_{1}^{2}\right)\right\rangle^{2 b}}\right)^{1 / 2}\right\|_{L_{\tau}^{\infty} L_{\xi}^{\infty}},
\end{gathered}
$$

where $\Omega_{I ; \xi}=\left\{\xi_{1}: 1 \leq\left|\xi-\xi_{1}\right|,\left|\xi-2 \xi_{1}\right| \geq|\xi|^{-3 / 2}\right\}$. Noting that

$$
2 b>1, \quad \sigma=\min \left\{\frac{s}{5}+\frac{1}{20}, \frac{3}{16}\right\}
$$


and using (3.3) and the change of variables

$$
\mu=5 \xi \xi_{1}\left(\xi-\xi_{1}\right)\left(\xi^{2}-\xi \xi_{1}+\xi_{1}^{2}\right)
$$

we have

$$
\begin{aligned}
& \left\|\left(\int_{\mathbb{R}} \frac{|\xi|^{11 / 2-8 \sigma}\left|\xi-\xi_{1}\right|^{1 / 2-2 s+8 \sigma} \chi_{\Omega_{I, \xi}}\left(\xi_{1}\right)}{\left(\left|\xi-\xi_{1}\right||\xi|^{4}\right)^{1-2 \sigma}\left\langle\tau-\xi^{5}+5 \xi \xi_{1}\left(\xi-\xi_{1}\right)\left(\xi^{2}-\xi \xi_{1}+\xi_{1}^{2}\right)\right\rangle^{2 b}} d \xi_{1}\right)^{1 / 2}\right\|_{L_{\tau}^{\infty} L_{\xi}^{\infty}} \\
& \quad=\left\|\left(\int_{\mathbb{R}} \frac{|\xi|^{3 / 2}\left|\xi-\xi_{1}\right|^{-1 / 2-2 s+10 \sigma} X \Omega_{\Omega_{I ; \xi}}\left(\xi_{1}\right)}{\left\langle\tau-\xi^{5}+5 \xi \xi_{1}\left(\xi-\xi_{1}\right)\left(\xi^{2}-\xi \xi_{1}+\xi_{1}^{2}\right)\right\rangle^{2 b}} d \xi_{1}\right)^{1 / 2}\right\|_{L_{\tau}^{\infty} L_{\xi}^{\infty}} \\
& \quad \leq\left\|\left(\int_{|\mu| \leq 3\left|\tau-\xi^{5}\right|} \frac{|\xi|^{3 / 2}}{\left\langle\tau-\xi^{5}+\mu\right\rangle^{2 b} 5|\xi|\left|\xi-2 \xi_{1}\right|\left|\xi^{2}-2 \xi_{1}+2 \xi_{1}^{2}\right|} d \mu\right)^{1 / 2}\right\|_{L_{\tau}^{\infty} L_{\xi}^{\infty}} \\
& \quad \leq\left(\frac{5}{2}\right)^{-1 / 2}\left\|\left(\int_{|\mu| \leq 3\left|\tau-\xi^{5}\right|} \frac{d \mu}{\left\langle\tau-\xi^{5}+\mu\right\rangle^{2 b}}\right)^{1 / 2}\right\|_{L_{\tau}^{\infty} L_{\xi}^{\infty}} \\
& \quad \leq C_{8,2 b}\left(\frac{5}{2}\right)^{-1 / 2},
\end{aligned}
$$

where we used the inequality

$$
5|\xi|\left|\xi-2 \xi_{1}\right|\left|\xi^{2}-2 \xi \xi_{1}+2 \xi_{1}^{2}\right| \geq \frac{5}{2}|\xi|^{3 / 4} \text { in } \Omega_{I ; \xi}
$$

which follows from

$$
\left|\xi^{2}-2 \xi \xi_{1}+2 \xi_{1}^{2}\right| \geq \frac{1}{2}|\xi|^{2}, \quad\left|\xi-2 \xi_{1}\right| \geq|\xi|^{-3 / 2}
$$

Thus, (3.27) is bounded by

$$
M_{5, s, b, b^{\prime}}=5^{3 / 2}\left(\frac{5}{16}\right)^{\left(b^{\prime}-b\right)-1 / 2} 2^{|s|} C_{9, s} \max \left\{C_{7,2 b, 2 b} C_{8,2 b}, C_{7,2\left(1-b^{\prime}\right), 2 b} 3^{b^{\prime}-1 / 2}\right\}
$$

in $\Omega_{2.1 .2}$. In the other regions, we can get bounds of (3.27) by a similar argument to (3.57)(3.63). Therefore we omit the proof. 
Now (3.27) are shown to be bounded. Therefore, combining (3.4), (3.6), (3.12), and (3.18)-(3.26) and setting

$$
C_{3, s, b, b^{\prime}}=M_{1, s, b, b^{\prime}}+7 M_{2, s, b, b^{\prime}}+2 M_{3, s, b, b^{\prime}}+3 M_{4, s, b, b^{\prime}}+17 M_{5, s, b, b^{\prime},}
$$

we have (2.17). This completes the proof of Lemma 2.4.

Remark 3.2. We briefly state the reason why the term $10 u \partial_{x}^{3} u$ is removed from $(1.1)_{2}$. In order to show the existence of the solution of $(1.1)_{2}$, we have to prove the following estimate:

$$
\left\|u \partial_{x}^{3} v\right\|_{X_{b^{\prime}-1}^{s}} \leq C_{3, s, b, b^{\prime}}\|u\|_{X_{b}^{s}}\|v\|_{X_{b}^{s}}
$$

Unfortunately, we are not able to prove it, because our method can be used to estimate $\partial_{x}^{l}\left(\partial_{x}^{m} u \partial_{x}^{n} v\right)$ in the case where $\operatorname{lm} n \geq 1$, but not in the case where $\operatorname{lm} n=0$. Therefore, it is necessarily for us to remove the term $10 u \partial_{x}^{3} u$ from $(1.1)_{2}$ unavoidably.

\section{Analyticity}

In this section we prove the analyticity of the solution $u \equiv u_{0}$ given in Proposition 2.1. The proof is established by Propositions 4.5-4.9. To prove these propositions we prepare four lemmas (Lemmas 4.1-4.4).

Lemma 4.1 (see [21]). Let

$$
s>-\frac{7}{4}, \quad b \in\left(\frac{1}{2}, \frac{1}{2}+\sigma\right)
$$

where $\sigma=\min \{1 / 4,(4 s+11) / 8,(s+6) / 5\}$. Then

$$
\left\|\partial_{x}(u v)\right\|_{X_{b-1}^{s}} \leq M_{6, s, b}\|u\|_{X_{b}^{s}}\|v\|_{X_{b}^{s}}
$$

where $M_{6, s, b}$ is a constant depending on $s$ and $b$.

Lemma 4.2 (see [7]). Let

$$
f \in H^{s}\left(\mathbb{R}^{n}\right), \quad g \in H^{r}\left(\mathbb{R}^{n}\right) .
$$

Suppose that

$$
0 \leq s, r \leq \frac{n}{2}, \quad \frac{n}{2} \leq s+r
$$

Then, for any $\sigma_{1}<s+r-n / 2$,

$$
\|f g\|_{H^{\sigma_{1}\left(\mathbb{R}^{n}\right)}} \leq M_{7}\|f\|_{H^{s}\left(\mathbb{R}^{n}\right)}\|g\|_{H^{r}\left(\mathbb{R}^{n}\right)}
$$

holds, where $M_{7}=M_{7, s, r, \sigma_{1}, n}$ is a constant depending on $s, r, \sigma_{1}$, and $n$. 
Lemma 4.3. Let $\left(t_{0}, x_{0}\right)$ be an arbitrarily fixed point in $\{(-T, 0) \cup(0, T)\} \times \mathbb{R}$.

(1) Suppose that $b \in(0,1], r \in(-\infty, 0]$. Then, for a sufficiently small $\varepsilon_{1}>0$ such that

$$
\begin{gathered}
\varepsilon_{1}= \begin{cases}\varepsilon^{4}, & \text { if }-\frac{9}{2}<r \leq 0, \\
\varepsilon^{\alpha} \quad \text { with } \alpha>-\frac{1}{2}-r, \text { if } r \leq-\frac{9}{2},\end{cases} \\
\left\|\left\langle D_{t, x}\right\rangle^{5 b} g\right\|_{L_{t}^{2}\left(\mathbb{R} ; H_{x}^{r}(\mathbb{R})\right)} \leq M_{8, r, b, \varepsilon_{1}}\left\{\|g\|_{X_{b-1}^{r}}+\left\|t \partial_{x}^{5} g\right\|_{X_{b-1}^{r}}+\left\|P^{5} g\right\|_{X_{b-1}^{r}}\right\}
\end{gathered}
$$

holds for all $g \in X_{b-1}^{r}$ satisfying

$$
\text { supp } g \subset B_{2 \varepsilon_{1}}\left(t_{0}, x_{0}\right), \quad t \partial_{x}^{5} g, P^{5} g \in X_{b-1}^{r},
$$

where $M_{8, r, b, \varepsilon_{1}}=M_{8, r, b,\left(t_{0}, x_{0}\right), \varepsilon_{1}}$ depends on $r, b,\left(t_{0}, x_{0}\right)$, and $\varepsilon$.

(2) Let $\mu>0$. Then, for a sufficiently small $\varepsilon_{2}=\varepsilon^{4}>0$,

$$
\left\|\left\langle D_{t, x}\right\rangle^{\mu} g\right\|_{L_{t}^{2} L_{x}^{2}} \leq M_{9, \mu, \varepsilon_{2}}\left\{\|g\|_{H_{t, x}^{\mu-5}\left(\mathbb{R}^{2}\right)}+\left\|t \partial_{x}^{5} g\right\|_{H_{t, x}^{\mu-5}\left(\mathbb{R}^{2}\right)}+\left\|P^{5} g\right\|_{H_{t, x}^{\mu-5}(\mathbb{R})}\right\}
$$

holds for all $g \in H_{t, x}^{\mu-5}\left(\mathbb{R}^{2}\right)$ satisfying

$$
\operatorname{supp} g \subset B_{2 \varepsilon_{2}}\left(t_{0}, x_{0}\right), \quad t \partial_{x}^{5} g, P^{5} g \in H_{t, x}^{\mu-5}\left(\mathbb{R}^{2}\right),
$$

where $M_{9, \mu, \varepsilon_{2}}=M_{9, \mu,\left(t_{0}, x_{0}\right), \varepsilon_{2}}$ depends on $\mu,\left(t_{0}, x_{0}\right)$, and $\varepsilon$.

Let $\rho(t, x)$ be a smooth cut-off function around the freezing point $\left(t_{0}, x_{0}\right)$ such that $\rho \in C_{0}^{\infty}\left(B_{2 \varepsilon^{4}}\left(t_{0}, x_{0}\right)\right)$.

Lemma 4.4. Let $s, b \in \mathbb{R}$. Then

$$
\|\rho f\|_{X_{b}^{s}} \leq M_{10, s, b, \rho, \varepsilon_{3}} \varepsilon_{3}{ }^{-|s|-9|b|}\|f\|_{X_{b}^{s+4|b|},}
$$

where $\varepsilon_{3}=\varepsilon^{4}$ and $M_{10, s, b, \rho, \varepsilon_{3}}=10^{|b| / 2}\left\|\left\langle\varepsilon_{3}^{4} \tau-\xi^{5}\right\rangle^{|b|}\langle\xi\rangle^{|s|+4|b|} \mathcal{F}_{t, x} \rho(\tau, \xi)\right\|_{L_{\tau}^{1} L_{\xi}^{1}}$.

Proof. Lemmas 4.3 and 4.4 are proved by the same method as Lemmas 3.2 and 5.2 in [7].

Proposition 4.5. Let $s>1 / 8$, and let $b \in(1 / 2,23 / 40)$. Then, for a sufficiently small $\varepsilon^{4}>0$, there exist positive constants $K_{1, \rho}$ and $A_{1}$ such that

$$
\left\|\rho P^{k} u\right\|_{H_{t, x}^{1 / 3}\left(\mathbb{R}^{2}\right)}+\left\|\rho P^{k} u\right\|_{L_{t}^{2}\left(\mathbb{R} ; H_{x}^{1}\right)} \leq K_{1, \rho} A_{1}^{k} k !
$$

holds for all $k=0,1,2, \ldots$, where $A_{1}=2 A_{0}^{-1}$ and $K_{1, \rho}=K_{1, s, b,\left(t_{0}, x_{0}\right), \varepsilon, \rho}$ is a constant depending on $s$, $b,\left(t_{0}, x_{0}\right), \varepsilon$ and $\rho$. 
Proof. By Plancherel Theorem and Lemma 4.3 with $g=\rho P^{k} u$, we have

$$
\begin{aligned}
& \left\|\rho P^{k} u\right\|_{H_{t, x}^{1 / 3}\left(\mathbb{R}^{2}\right)}+\left\|\rho P^{k} u\right\|_{L_{t}^{2}\left(\mathbb{R} ; H_{x}^{1}\right)} \\
& \quad \leq \frac{2}{(2 \pi)^{2}}\left\|\left\langle D_{t, x}\right\rangle^{5 b} \rho P^{k} u\right\|_{L_{t}^{2}\left(\mathbb{R} ; H_{x}^{r}(\mathbb{R})\right)} \\
& \quad \leq \frac{2}{(2 \pi)^{2}} M_{8, r, b, \varepsilon^{4}}\left\{\left\|\rho P^{k} u\right\|_{X_{b-1}^{r}}+\left\|t \partial_{x}^{5}\left(\rho P^{k} u\right)\right\|_{X_{b-1}^{r}}+\left\|P^{5}\left(\rho P^{k} u\right)\right\|_{X_{b-1}^{r}}\right\},
\end{aligned}
$$

where

$$
\begin{aligned}
& r=s-2, \quad \text { if } \frac{1}{8}<s \leq 2, \\
& -\frac{15}{8}<r \leq 0, \quad \text { if } s>2 .
\end{aligned}
$$

We note that $r \leq s-2$ holds. Put $K_{2, s, b}=\left|\|\mathbf{u} \mid\|_{\mathscr{A}_{A_{0}}\left(X_{b}^{s}\right)}\right.$. Since (2.8) and Remark 2.2 yield

$$
\left\|P^{k} u\right\|_{X_{b}^{s}} \leq K_{2, s, b}\left(A_{0}^{-1}\right)^{k} k !, \quad k=0,1,2, \ldots,
$$

it follows from Lemma 4.4 that

$$
\begin{aligned}
& \left\|\rho P^{k} u\right\|_{X_{b-1}^{r}} \leq M_{10, r, b-1, \rho, \varepsilon^{4}} \mathcal{E}^{-4|r|-36|b-1|}\left\|P^{k} u\right\|_{X_{b-1}^{s}} \\
& \leq K_{2, s, b-1} M_{10, r, b-1, \rho, \varepsilon^{4}} \mathcal{E}^{-4|r|-36|b-1|}\left(A_{0}^{-1}\right)^{k} k !, \\
& \left\|P^{5}\left(\rho P^{k} u\right)\right\|_{X_{b-1}^{r}} \leq \sum_{l=0}^{5} \frac{5 !}{(5-l) ! l !}\left\|P^{5-l} \rho P^{l+k} u\right\|_{X_{b-1}^{r}} \\
& \leq \sum_{l=0}^{5} \frac{5 !}{(5-l) ! l !} M_{10, r, b-1, \rho_{l}, \varepsilon^{4}} \mathcal{E}^{-4|r|-36|b-1|}\left\|P^{l+k} u\right\|_{X_{b-1}^{s}} \\
& \leq \max _{0 \leq l \leq 5} M_{10, r, b-1, \rho_{l}, \varepsilon^{4}} \mathcal{E}^{-4|r|-36|b-1|} K_{2, s, b-1} \sum_{l=0}^{5} \frac{5 !}{(5-l) ! l !} \frac{(k+l) !}{2^{k} k !}\left(A_{0}^{-1}\right)^{l}\left(2 A_{0}^{-1}\right)^{k} k ! \\
& \leq K_{3} A_{1}^{k} k !,
\end{aligned}
$$

where $\rho_{l}=P^{5-l} \rho_{,} K_{3}$ is some constant and $A_{1}=\left(2 A_{0}^{-1}\right)$. 
Now we estimate $\left\|t \partial_{x}^{5}\left(\rho P^{k} u\right)\right\|_{X_{b-1}^{r}}$. By using

$$
\begin{gathered}
t \partial_{x}^{5}\left(\rho P^{k} u\right)=t \rho\left(\partial_{x}^{5} P^{k} u\right)+5 t \partial_{x}^{2}\left(\left(\partial_{x}^{2} \rho\right)\left(\partial_{x} P^{k} u\right)\right)+5 t \partial_{x}\left(\left(\partial_{x} \rho\right)\left(\partial_{x}^{3} P^{k} u\right)\right)+t\left(\partial_{x}^{5} \rho\right) P^{k} u \\
\left(\partial_{x}^{5} P^{k} u\right)=-\frac{1}{5}\left\{P^{k+1} u-x \partial_{x} P^{k} u\right\}+t N_{k}(u)
\end{gathered}
$$

we have

$$
\begin{aligned}
\left\|t \partial_{x}^{5}\left(\rho P^{k} u\right)\right\|_{X_{b-1}^{r}} \leq & \frac{1}{5}\left\{\left\|\rho P^{k+1} u\right\|_{X_{b-1}^{r}}+\left\|\rho x \partial_{x} P^{k} u\right\|_{X_{b-1}^{r}}\right\}+\left\|t \rho N_{k}(u)\right\|_{X_{b-1}^{r}} \\
& +5\left\|\partial_{x}^{2}\left(t\left(\partial_{x}^{2} \rho\right)\left(\partial_{x} P^{k} u\right)\right)\right\|_{X_{b-1}^{r}}+5\left\|\partial_{x}\left(t\left(\partial_{x} \rho\right)\left(\partial_{x}^{3} P^{k} u\right)\right)\right\|_{X_{b-1}^{r}} \\
& +\left\|t\left(\partial_{x}^{5} \rho\right) P^{k} u\right\|_{X_{b-1}^{r}}
\end{aligned}
$$

In the same manner as (4.15), we have

$$
\begin{aligned}
& \left\|\rho P^{k+1} u\right\|_{X_{b-1}^{r}} \leq M_{10, r, b-1, \rho, \varepsilon^{4}} \mathcal{E}^{-4|r|-36|b-1|}\left\|P^{k+1} u\right\|_{X_{b-1}^{s}}
\end{aligned}
$$

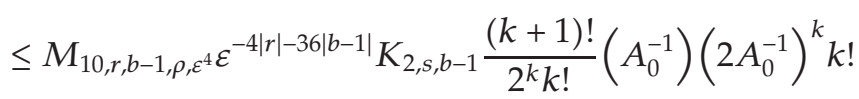

$$
\begin{aligned}
& \leq K_{4} A_{1}^{k} k !
\end{aligned}
$$

where $K_{4}$ is some constant. By Lemmas 4.1 and 4.4 , we have

$$
\begin{aligned}
& \left\|\rho x \partial_{x} P^{k} u\right\|_{X_{b-1}^{r}} \leq\left\|\partial_{x}\left(\rho x P^{k} u\right)\right\|_{X_{b-1}^{r}}+\left\|\left(\partial_{x}(\rho x)\right) P^{k} u\right\|_{X_{b-1}^{r}} \\
& \leq M_{6, r, b}\|\rho x\|_{X_{b}^{s-2}}\left\|P^{k} u\right\|_{X_{b}^{s-2}}+M_{10, r, b-1, \partial_{x}(\rho x), \varepsilon^{4}} \varepsilon^{-4|r|-36|b-1|}\left\|P^{k} u\right\|_{X_{b-1}^{s}}
\end{aligned}
$$

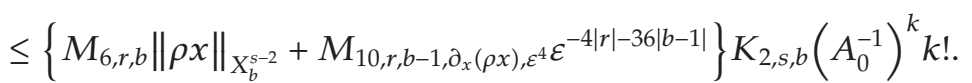


By Lemmas 2.4, 2.5, and 4.4, we have

$$
\begin{aligned}
& \left\|t \rho \mathcal{N}_{k}(u)\right\|_{X_{b-1}^{r}} \\
& \leq M_{10, r, b-1, t \rho, \varepsilon^{4}} \mathcal{E}^{-4|r|-36|b-1|} \\
& \times\left\{C_{4, s, b} \sum_{\mathbf{k}} \frac{k ! 4^{k_{4}}}{k_{1} ! k_{2} ! k_{3} ! k_{4} !}\left\|P^{k_{1}} u\right\|_{X_{b}^{s}}\left\|P^{k_{2}} u\right\|_{X_{b}^{s}}\left\|P^{k_{3}} u\right\|_{X_{b}^{s}}\right. \\
& \left.+C_{3, s, b} \sum_{\mathbf{k}} \frac{k ! 3^{k_{4}}}{k_{1} ! k_{2} ! k_{3} ! k_{4} !}\left\|P^{k_{1}} u\right\|_{X_{b}^{s}}\left\|P^{k_{2}} u\right\|_{X_{b}^{s}}\right\} \\
& \leq M_{10, r, b-1, t \rho, \varepsilon^{4}} \mathcal{E}^{-4|r|-36|b-1|} \\
& \times\left\{C_{4, s, b} K_{2, s, b}^{3} \sum_{\mathbf{k}} \frac{4^{k_{4}}}{k_{4} !}\left(A_{0}^{-1}\right)^{k_{1}+k_{2}+k_{3}}+C_{3, s, b} K_{2, s, b}^{2} \sum_{\mathbf{k}} \frac{3^{k_{4}}}{k_{3} ! k_{4} !}\left(A_{0}^{-1}\right)^{k_{1}+k_{2}}\right\} k ! \\
& \leq M_{10, r, b-1, t \rho, \varepsilon^{4}} \mathcal{E}^{-4|r|-36|b-1|} e^{4 / A_{0}^{-1}}\left\{C_{4, s, b} K_{2, s, b}^{3} \frac{(k+1)}{2^{k}}+C_{3, s, b} K_{2, s, b}^{2} \frac{(k+1)(k+2)}{2^{k}}\right\}\left(2 A_{0}^{-1}\right)^{k} k ! \\
& \leq K_{5} A_{1}^{k} k !
\end{aligned}
$$

where $K_{5}$ is some constant. We also have

$$
\begin{aligned}
\left\|\partial_{x}^{2}\left(t\left(\partial_{x}^{2} \rho\right)\left(\partial_{x} P^{k} u\right)\right)\right\|_{X_{b-1}^{r}} & \leq\left\|\partial_{x}\left(t\left(\partial_{x}^{2} \rho\right)\left(\partial_{x} P^{k} u\right)\right)\right\|_{X_{b-1}^{s-1}} \\
& \leq C_{3, s-1, b}\left\|t \partial_{x} \rho\right\|_{X_{b}^{s-1}}\left\|P^{k} u\right\|_{X_{b}^{s-1}} \\
& \leq C_{3, s-1, b} K_{2, s-1, b}\left\|t \partial_{x} \rho\right\|_{X_{b}^{s-1}}\left(A_{0}^{-1}\right)^{k} k !, \\
\left\|\sigma_{x}\left(t\left(\partial_{x} \rho\right)\left(\partial_{x}^{3} P^{k} u\right)\right)\right\|_{X_{b-1}^{r}} & \leq C_{3, s-2, b}\|t \rho\|_{X_{b}^{s-2}}\left\|\partial_{x}^{2} P^{k} u\right\|_{X_{b}^{s-2}} \\
& \leq C_{3, s-2, b}\|t \rho\|_{X_{b}^{s-2}}\left\|P^{k} u\right\|_{X_{b}^{s}} \\
& \leq C_{3, s-2, b} K_{2, s, b}\|t \rho\|_{X_{b}^{s-2}}\left(A_{0}^{-1}\right)^{k} k ! .
\end{aligned}
$$

In the same manner as (4.15), we have

$$
\left\|t\left(\partial_{x}^{5} \rho\right) P^{k} u\right\|_{X_{b-1}^{r}} \leq M_{10, r, b-1, t \partial_{x}^{5} \rho, \varepsilon^{4}} K_{2, s, b-1}\left(A_{0}^{-1}\right)^{k} k ! .
$$

Hence

$$
\left\|t \partial_{x}^{5}\left(\rho P^{k} u\right)\right\|_{X_{b-1}^{r}} \leq K_{6} A_{1}^{k} k !
$$


where $K_{6}$ is some constant. Putting

$$
K_{1, \rho}=\frac{3}{2 \pi^{2}} M_{8, r, b, \varepsilon^{4}} \max \left\{K_{2, s, b-1} M_{10, r, b-1, \rho, \varepsilon^{4}} \mathcal{E}^{-4|r|-36|b-1|}, K_{3}, K_{6}\right\},
$$

we have (4.11).

Proposition 4.6. Under the same assumption as in Proposition 4.5, there exist $K_{7}$ and $A_{2}$ such that

$$
\left\|\rho_{4} P^{k} u\right\|_{H_{t, x}^{11 / 2}(\mathbb{R})} \leq K_{7} A_{2}^{k} k !
$$

holds for all $k=0,1,2, \ldots$, where $\rho_{4}$ is a smooth cut-off function such that

$$
\begin{gathered}
\rho_{4} \leq \min \left\{\rho, \rho^{4}\right\}, \\
\rho_{4} \equiv 1 \quad \text { on }\left(t_{0}-\varepsilon^{4}, t_{0}+\varepsilon^{4}\right) \times\left(x_{0}-\varepsilon^{4}, x_{0}+\varepsilon^{4}\right) .
\end{gathered}
$$

Proof. At first, we show that there exists a constant $K_{7,1 / 2}$ and $A_{3}$ such that

$$
\left\|\rho_{4} P^{k} u\right\|_{H_{t, x}^{1 / 2}\left(\mathbb{R}^{2}\right)} \leq K_{7,1 / 2} A_{3}^{k} k !, \quad k=0,1,2, \ldots
$$

Applying Lemma 4.3 with $\mu=1 / 2$ and $g=\rho_{4} P^{k} u$, we have

$$
\begin{aligned}
& \left\|\left\langle D_{t, x}\right\rangle^{1 / 2} \rho_{4} P^{k} u\right\|_{L_{t}^{2} L_{x}^{2}} \\
& \quad \leq M_{9,1 / 2, \varepsilon^{4}}\left\{\left\|\rho_{4} P^{k} u\right\|_{H_{t, x}^{-9 / 2}\left(\mathbb{R}^{2}\right)}+\left\|t \partial_{x}^{5}\left(\rho_{4} P^{k} u\right)\right\|_{H_{t, x}^{-9 / 2}\left(\mathbb{R}^{2}\right)}+\left\|P^{5}\left(\rho_{4} P^{k} u\right)\right\|_{H_{t, x}^{-9 / 2}\left(\mathbb{R}^{2}\right)}\right\} .
\end{aligned}
$$

By Proposition 4.5, we have

$$
\begin{aligned}
\left\|\rho_{4} P^{k} u\right\|_{H_{t, x}^{-9 / 2}\left(\mathbb{R}^{2}\right)} & \leq K_{1, \rho_{4}} A_{1}^{k} k ! \\
\left\|P^{5}\left(\rho_{4} P^{k} u\right)\right\|_{H_{t, x}^{-9 / 2}\left(\mathbb{R}^{2}\right)} & \leq \sum_{l=0}^{5} \frac{5 !}{l !(5-l) !}\left\|P^{5-l} \rho_{4}\right\|_{L_{t, x}^{\infty}\left(\mathbb{R}^{2}\right)}\left\|\rho P^{k+l} u\right\|_{L_{t, x}^{2}\left(\mathbb{R}^{2}\right)} \\
& \leq K_{1, \rho} \max _{0 \leq l \leq 5}\left\|P^{5-l} \rho_{4}\right\|_{L_{t, x}^{\infty}\left(\mathbb{R}^{2}\right)} \sum_{l=0}^{5} \frac{5 !}{(5-l) ! l !} \frac{(k+l) !}{2^{k} k !}\left(A_{1}^{-1}\right)^{l}\left(2 A_{1}^{-1}\right)^{k} k ! \\
& \leq K_{1, \rho} K_{8}\left(2 A_{1}^{-1}\right)^{k} k !,
\end{aligned}
$$

where $K_{8}$ is some constant. 
30

Advances in Mathematical Physics

Now we estimate $\left\|t \partial_{x}^{5}\left(\rho_{4} P^{k} u\right)\right\|_{H_{t, x}^{-9 / 2}\left(\mathbb{R}^{2}\right)}$. Using (4.18) and

$$
\partial_{x}^{5}\left(\rho_{4} f\right)=\rho_{4} \partial_{x}^{5} f+\sum_{l=1}^{5}(-1)^{l-1} \frac{5 !}{l !(5-l) !} \partial_{x}^{5-l}\left(\left(\partial_{x}^{l} \rho_{4}\right) P^{k} u\right)
$$

we obtain

$$
\begin{aligned}
\left\|t \partial_{x}^{5}\left(\rho_{4} P^{k} u\right)\right\|_{H_{t, x}^{-9 / 2}\left(\mathbb{R}^{2}\right)} \leq & \frac{1}{5}\left\{\left\|\rho_{4} P^{k+1} u\right\|_{H_{t, x}^{-9 / 2}\left(\mathbb{R}^{2}\right)}+\left\|\rho_{4} x \partial_{x} P^{k} u\right\|_{H_{t, x}^{-9 / 2}\left(\mathbb{R}^{2}\right)}\right\} \\
& +\left\|t \rho_{4} \mathcal{N}_{k}(u)\right\|_{H_{t, x}^{-9 / 2}\left(\mathbb{R}^{2}\right)}+\sum_{l=1}^{5} \frac{5 !}{l !(5-l) !}\left\|t \partial_{x}^{5-l}\left(\left(\partial_{x}^{l} \rho_{4}\right) P^{k} u\right)\right\|_{H_{t, x}^{-9 / 2}\left(\mathbb{R}^{2}\right)} .
\end{aligned}
$$

By Proposition 4.5, we have

$$
\begin{aligned}
\frac{1}{5}\left\|\rho_{4} P^{k+1} u\right\|_{H_{t, x}^{-9 / 2}\left(\mathbb{R}^{2}\right)} & \leq \frac{1}{5}\left\|\rho_{4}\right\|_{L_{t}^{\infty} L_{x}^{\infty}}\left\|\rho P^{k+1} u\right\|_{L_{t}^{2} L_{x}^{2}} \\
& \leq \frac{1}{5} K_{1, \rho}\left\|\rho_{4}\right\|_{L_{t}^{\infty} L_{x}^{\infty}} \frac{(k+1) !}{2^{k} k !}\left(A_{1}^{-1}\right)\left(2 A_{1}^{-1}\right)^{k} k ! \\
& \leq K_{1, \rho} K_{9}\left(2 A_{1}^{-1}\right)^{k} k !, \\
\frac{1}{5}\left\|\rho_{4} x \partial_{x} P^{k} u\right\|_{H_{t, x}^{-9 / 2}\left(\mathbb{R}^{2}\right)} & \leq \frac{1}{5}\left(\left\|x \rho_{4}\right\|_{L_{t}^{\infty} L_{x}^{\infty}}+\left\|\partial_{x}\left(x \rho_{4}\right)\right\|_{L_{t}^{\infty} L_{x}^{\infty}}\right)\left\|\rho P^{k} u\right\|_{L_{t}^{2} L_{x}^{2}} \\
& \leq \frac{1}{5}\left(\left\|x \rho_{4}\right\|_{L_{t}^{\infty} L_{x}^{\infty}}+\left\|\partial_{x}\left(x \rho_{4}\right)\right\|_{L_{t}^{\infty} L_{x}^{\infty}}\right) K_{1, \rho} A_{1}^{k} k !,
\end{aligned}
$$

where $K_{9}$ is some constant. By Sobolev embedding theorem and Proposition 4.5, we have

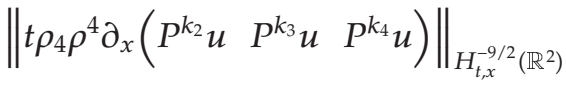

$$
\begin{aligned}
& \leq\left\|\langle|\tau|+|\xi|\rangle^{-7 / 2}\right\|_{L_{\tau}^{2} L_{\xi}^{2}}\left(\left\|\partial_{x} t \rho_{4}\right\|_{L_{t}^{\infty} L_{x}^{\infty}}+\left\|t \rho_{4}\right\|_{L_{t}^{\infty} L_{x}^{\infty}}\right)\|\rho\|_{L_{t}^{\infty} L_{x}^{\infty}} \\
& \times\left\|\rho P^{k_{2}} u\right\|_{L_{t}^{3} L_{x}^{3}}\left\|\rho P^{k_{3}} u\right\|_{L_{t}^{3} L_{x}^{3}}\left\|\rho P^{k_{4}} u\right\|_{L_{t}^{3} L_{x}^{3}} \\
& \leq K_{10,-7 / 2}\|\rho\|_{L_{t}^{\infty} L_{x}^{\infty}}\left\|\rho P^{k_{2}} u\right\|_{H_{t, x}^{1 / 3}\left(\mathbb{R}^{2}\right)}\left\|\rho P^{k_{3}} u\right\|_{H_{t, x}^{1 / 3}\left(\mathbb{R}^{2}\right)}\left\|\rho P^{k_{4}} u\right\|_{H_{t, x}^{1 / 3}\left(\mathbb{R}^{2}\right)} \\
& \leq K_{10,-7 / 2}\|\rho\|_{L_{t}^{\infty} L_{x}^{\infty}} K_{1, \rho}^{3} A_{1}^{k_{2}+k_{3}+k_{4}} k_{2} ! k_{3} ! k_{4} !
\end{aligned}
$$


Advances in Mathematical Physics

$$
\begin{aligned}
& \left.\| t \rho_{4} \rho^{4} \partial_{x}\left(\left(\partial_{x} P^{k_{1}} u\right) \partial_{x} P^{k_{2}} u\right)\right) \|_{H_{t, x}^{-9 / 2}\left(\mathbb{R}^{2}\right)} \\
& \quad \leq K_{10,-7 / 2}\left\|\rho^{2} \partial_{x} P^{k_{1}} u\right\|_{L_{t}^{2} L_{x}^{2}}\left\|\rho^{2} \partial_{x} P^{k_{2}} u\right\|_{L_{t}^{2} L_{x}^{2}} \\
& \quad \leq K_{10,-7 / 2}\left(\left\|\partial_{x} \rho\right\|_{L_{t}^{\infty} L_{x}^{\infty}}+\|\rho\|_{L_{t}^{\infty} L_{x}^{\infty}}\right)^{2}\left\|\rho P^{k_{1}} u\right\|_{L_{t}^{2}\left(\mathbb{R} ; H_{x}^{1}\right)}\left\|\rho P^{k_{2}} u\right\|_{L_{t}^{2}\left(\mathbb{R} ; H_{x}^{1}\right)} \\
& \quad \leq K_{11,-7 / 2} K_{1, \rho}^{2} A_{1}^{k_{1}+k_{2}} k_{1} ! k_{2} !,
\end{aligned}
$$

where $K_{10,-7 / 2}, K_{11,-7 / 2}$ are some constants. Therefore, we have by (4.35)

$$
\begin{aligned}
& \left\|t \rho_{4} \mathcal{N}_{k}(u)\right\|_{H_{t, x}^{-9 / 2}\left(\mathbb{R}^{2}\right)} \\
& \leq\left\|t \rho_{4} \rho^{4} \mathcal{N}_{k}(u)\right\|_{H_{t, x}^{-9 / 2}\left(\mathbb{R}^{2}\right)} \\
& \left.\leq \sum_{\mathbf{k}} \frac{k ! 4^{k_{1}}}{k_{1} ! k_{2} ! k_{3} ! k_{4} !} \| t \rho_{4} \rho^{4} \partial_{x}\left(P^{k_{2}} u P^{k_{3}} u P^{k_{4}} u\right)\right) \|_{H_{t, x}^{-9 / 2}\left(\mathbb{R}^{2}\right)} \\
& \quad+\sum_{\mathbf{k}} \frac{k ! 3 k^{k_{4}}}{k_{1} ! k_{2} ! k_{3} ! k_{4} !}\left\|t \rho_{4} \rho^{4} \partial_{x}\left(\left(\partial_{x} P^{k_{2}} u\right)\left(\partial_{x} P^{k_{1}} u\right)\right)\right\|_{H_{t, x}^{-9 / 2}\left(\mathbb{R}^{2}\right)} \\
& \leq K_{10,-7 / 2}\|\rho\|_{L_{t}^{\infty} L_{x}^{\infty}} K_{1, \rho}^{3} k ! \sum_{\mathbf{k}} \frac{4^{k_{4}}}{k_{4} !} A_{1}^{k_{1}+k_{2}+k_{3}}+K_{11,-7 / 2} K_{1, \rho}^{2} k ! \sum_{\mathbf{k}} \frac{3^{k_{4}}}{k_{3} ! K_{4} !} A_{1}^{k_{1}+k_{2}} \\
& \leq \\
& \leq
\end{aligned}
$$

By Proposition 4.5, we have

$$
\begin{aligned}
\sum_{l=1}^{5} \frac{5 !}{l !(5-l) !}\left\|\partial_{x}^{5-l}\left(\left(t \partial_{x}^{l} \rho_{4}\right) P^{k} u\right)\right\|_{H_{t, x}^{-9 / 2}\left(\mathbb{R}^{2}\right)} & \leq \sum_{l=1}^{5} \frac{5 !}{l !(5-l) !}\left\|t \partial_{x}^{l} \rho_{4}\right\|_{L_{t}^{\infty} L_{x}^{\infty}}\left\|\rho P^{k} u\right\|_{L_{t}^{2} L_{x}^{2}} \\
& \leq K_{12} K_{1, \rho} A_{1}^{k} k !,
\end{aligned}
$$

where $K_{12}$ is some constant. Hence

$$
\left\|t \partial_{x}^{5}\left(\rho_{4} P^{k} u\right)\right\|_{H_{t, x}^{-9 / 2}\left(\mathbb{R}^{2}\right)} \leq K_{13, K_{1, \rho}, K_{10,-7 / 2}} \max \left\{2 A_{1}^{-1}, A_{1}\right\}^{k} k !,
$$

where $K_{13, K_{1, \rho}, K_{10,-7 / 2}}$ is some constant. Putting $A_{3}=\max \left\{2 A_{1}^{-1}, A_{1}\right\}$,

$$
K_{7,1 / 2}=K_{7,1 / 2, K_{1, \rho}, K_{10,-7 / 2}}=3 M_{9,1 / 2, \varepsilon^{4}} \max \left\{K_{1, \rho_{4}}, K_{1, \rho} K_{8}, K_{14, K_{1, \rho}, K_{10,-7 / 2}}\right\},
$$


we have (4.29). Similarly, we can prove by (4.8) with $\mu=3 / 2$ and (4.29)

$$
\left\|\rho_{4} P^{k} u\right\|_{H_{t, x}^{3 / 2}\left(\mathbb{R}^{2}\right)} \leq K_{7,3 / 2, K_{7,1 / 2}, K_{10,-5 / 2}} A_{4}^{k} k !
$$

where $A_{4}=\max \left\{2 A_{3}^{-1}, A_{3}\right\}$. By (4.8) with $\mu=5 / 2$ and (4.40), we have

$$
\left\|\rho_{4} P^{k} u\right\|_{H_{t, x}^{5 / 2}\left(\mathbb{R}^{2}\right)} \leq K_{7,5 / 2, K_{7,3 / 2}, K_{10,0}} A_{5}^{k} k !
$$

where $A_{5}=\max \left\{2 A_{4}^{-1}, A_{4}\right\}$ and $K_{10,0}=\left(\left\|\partial_{x} t \rho_{4}\right\|_{L_{t}^{\infty} L_{x}^{\infty}}+\left\|t \rho_{4}\right\|_{L_{t}^{\infty} L_{x}^{\infty}}\right)\|\rho\|_{L_{t}^{\infty} L_{x}^{\infty}}$. Repeating the same method as in above, we obtain

$$
\begin{gathered}
\left\|\rho_{4} P^{k} u\right\|_{H_{t, x}^{7 / 2}\left(\mathbb{R}^{2}\right)} \leq K_{7,7 / 2, K_{8,5 / 2}, M_{7}} A_{6}^{k} k !, \\
\left\|\rho_{4} P^{k} u\right\|_{H_{t, x}^{9 / 2}\left(\mathbb{R}^{2}\right)} \leq K_{7,9 / 2, K_{8,7 / 2}, M_{7}} A_{7}^{k} k !, \\
\left\|\rho_{4} P^{k} u\right\|_{H_{t, x}^{11 / 2}\left(\mathbb{R}^{2}\right)} \leq K_{7,11 / 2, K_{8,9 / 2,}, K_{10,0}} A_{2}^{k} k !,
\end{gathered}
$$

where $A_{6}=\max \left\{2 A_{5}^{-1}, A_{5}\right\}, A_{7}=\max \left\{2 A_{6}^{-1}, A_{6}\right\}$ and $A_{2}=\max \left\{2 A_{7}^{-1}, A_{7}\right\}$. Putting $K_{7}=$ $K_{7,11 / 2, K_{8,9 / 2}, K_{10,0},}$ we have (4.27).

Remark 4.7. When $\mu=7 / 2, \mu=9 / 2$, we can obtain the similar estimates to (4.35) by using Lemma 4.2 and Sobolev embedding theorem.

Proposition 4.8. Suppose that (4.27) holds for all $k=0,1,2, \ldots$ Then

$$
\sup _{t \in I_{t_{0}}}\left\|\left(t^{1 / 5} \partial_{x}\right)^{l} P^{k} u\right\|_{H^{1}\left(I_{x_{0}}\right)} \leq K_{7} A_{8}^{k+l}(k+l) !
$$

holds for all $k, l=0,1,2, \ldots$, where

$$
\begin{gathered}
I_{t_{0}}=\left(t_{0}-\varepsilon^{4}, t_{0}+\varepsilon^{4}\right), \quad I_{x_{0}}=\left(x_{0}-\varepsilon^{4}, x_{0}+\varepsilon^{4}\right) \\
A_{8} \geq \max \left\{\left(\left|t_{0}\right|+\varepsilon^{4}\right)^{1 / 5}, A_{2},\left(\left|x_{0}\right|+\varepsilon^{4}+1\right)\left(\left|t_{0}-\varepsilon^{4}\right|\right)^{-1 / 5}+1\right. \\
\left.\left(\left|t_{0}\right|+\varepsilon^{4}\right)^{4 / 5} K_{7} e^{4 / A_{8}} \max \left\{\left|t_{0}-\varepsilon^{4}\right|^{-2 / 5}, K_{7}\right\}\right\} .
\end{gathered}
$$


Proof. We prove (4.43) by induction on $l$. When $l=0,1,2,3,4$, we use the trace theorem and (4.27) to obtain

$$
\begin{aligned}
\sup _{t \in I_{t_{0}}}\left\|\left(t^{1 / 5} \partial_{x}\right)^{l} P^{k} u\right\|_{H^{1}\left(I_{x_{0}}\right)} & \leq\left(\left|t_{0}\right|+\varepsilon^{4}\right)^{l / 5}\left\|\partial_{x}^{l} P^{k} u\right\|_{H_{t, x}^{3 / 2}\left(I_{t_{0}} \times I_{x_{0}}\right)} \\
& \leq\left(\left|t_{0}\right|+\varepsilon^{4}\right)^{l / 5}\left\|\rho_{4} P^{k} u\right\|_{H_{t, x}^{11 / 2}\left(\mathbb{R}^{2}\right)} \\
& \leq K_{7}\left(\left|t_{0}\right|+\varepsilon^{4}\right)^{l / 5} A_{2}^{k} k ! \\
& \leq K_{7} A_{8}^{k+l} k ! .
\end{aligned}
$$

We assume that (4.43) holds for any $l \geq 5$. Now we will prove

$$
\sup _{t \in I_{t_{0}}}\left\|\left(t^{1 / 5} \partial_{x}\right)^{l+1} P^{k} u\right\|_{H^{1}\left(I_{x_{0}}\right)} \leq K_{7} A_{8}^{k+l+1}(k+l+1) !
$$

Since

$$
\begin{aligned}
\left(t^{1 / 5} \partial_{x}\right)^{l} P^{k} u & =t^{(l-5) / 5} \partial_{x}^{l-5}\left(t \partial_{x}^{5} P^{k} u\right) \\
& =-\frac{1}{5} t^{(l-5) / 5} \partial_{x}^{l-5}\left\{P^{k+1} u-x \partial_{x} P^{k} u\right\}+\left(t^{1 / 5}\right)^{l} \partial_{x}^{l-5} \mathcal{N}_{k}(u)
\end{aligned}
$$

we have

$$
\begin{aligned}
\sup _{t \in I_{t_{0}}}\left\|\left(t^{1 / 5} \partial_{x}\right)^{l+1} P^{k} u\right\|_{H^{1}\left(I_{x_{0}}\right)} \leq & \frac{1}{5}\left\|t^{(l-4) / 5} \partial_{x}^{l-4} P^{k+1} u(t)\right\|_{H^{1}\left(I_{x_{0}}\right)} \\
& +\frac{1}{5}\left\|t^{(l-4) / 5} \partial_{x}^{l-4} x \partial_{x}\left(P^{k} u\right)\right\|_{H^{1}\left(I_{x_{0}}\right)}+\left\|\left(t^{1 / 5}\right)^{l+1} \partial_{x}^{l-4} N_{k}(u)\right\|_{H^{1}\left(I_{x_{0}}\right)} .
\end{aligned}
$$

By (4.43), we have

$$
\frac{1}{5}\left\|t^{(l-4) / 5} \partial_{x}^{l-4} P^{k+1} u(t)\right\|_{H^{1}\left(I_{x_{0}}\right)} \leq \frac{1}{5} K_{7} A_{8}^{k+l-3}(k+l-3) ! .
$$

Since

$$
t^{(l-4) / 5} \partial_{x}^{l-4}\left(x \partial_{x}\right)=x \partial_{x} t^{(l-4) / 5} \partial_{x}^{l-4}+(l-4) t^{(l-4) / 5} \partial_{x}^{l-4} \quad(l=5,6,7, \ldots),
$$


we have by (4.43)

$$
\begin{aligned}
\frac{1}{5} \| & t^{(l-4) / 5} \partial_{x}^{l-4} x \partial_{x}\left(P^{k} u\right) \|_{H^{1}\left(I_{x_{0}}\right)} \\
& \leq \frac{1}{5}\left\{\left\|x \partial_{x} t^{(l-4) / 5} \partial_{x}^{l-4} P^{k} u\right\|_{H^{1}\left(I_{x_{0}}\right)}+(l-4)\left\|t^{(l-4) / 5} \partial_{x}^{l-4} P^{k} u\right\|_{H^{1}\left(I_{x_{0}}\right)}\right\} \\
& \leq \frac{1}{5}\left(\left|x_{0}\right|+\varepsilon^{4}+1\right)\left|t_{0}-\varepsilon^{4}\right|^{-1 / 5}\left\|t^{(l-3) / 5} \partial_{x}^{l-3} P^{k} u\right\|_{H^{1}\left(I_{x_{0}}\right)}+\frac{1}{5}(l-4) K_{7} A_{8}^{k+l-4}(k+l-4) ! \\
& \leq \frac{1}{5}\left(\left|x_{0}\right|+\varepsilon^{4}+1\right)\left|t_{0}-\varepsilon^{4}\right|^{-1 / 5} K_{7} A_{8}^{k+l-3}(k+l-3) !+\frac{1}{5} K_{7} A_{8}^{k+l-3}(k+l-3) ! \\
& \leq \frac{1}{5} K_{7} A_{8}^{k+l-2}(k+l-3) !
\end{aligned}
$$

Now we estimate $\left\|\left(t^{1 / 5}\right)^{l+1} \partial_{x}^{l-4} \mathcal{N}_{k}(u)\right\|_{H^{1}\left(I_{x_{0}}\right)}$. We have

$$
\begin{aligned}
\left\|\left(t^{1 / 5}\right)^{l+1} \partial_{x}^{l-4} \mathcal{N}_{k}(u)\right\|_{H^{1}\left(I_{x_{0}}\right)} \leq & \left(\left|t_{0}\right|+\varepsilon^{4}\right)^{4 / 5} \\
& \times\left\{\sum_{\mathbf{k}} \frac{k ! 4^{k_{4}}}{k_{1} ! k_{2} ! k_{3} ! k_{4} !}\left\|t^{(l-3) / 5} \partial_{x}^{l-3}\left(P^{k_{1}} u P^{k_{2}} u P^{k_{3}} u\right)\right\|_{H^{1}\left(I_{x_{0}}\right)}\right. \\
& \left.+\sum_{\mathbf{k}} \frac{k ! 3^{k_{4}}}{k_{1} ! k_{2} ! k_{3} ! k_{4} !}\left\|t^{(l-3) / 5} \partial_{x}^{l-3}\left(\partial_{x} P^{k_{1}} u \partial_{x} P^{k_{2}} u\right)\right\|_{H^{1}\left(I_{x_{0}}\right)}\right\} .
\end{aligned}
$$

Since

$$
\sum_{1} \sum_{\mathbf{k}} \frac{\left(4 A_{8}^{-1}\right)^{k_{4}}}{k_{4} !} \frac{\left(l_{1}+k_{1}\right) !}{k_{1} ! l_{1} !} \frac{\left(l_{2}+k_{2}\right) !}{k_{2} ! l_{2} !} \frac{\left(l_{3}+k_{3}\right) !}{k_{3} ! l_{3} !} \frac{(l-3) ! k !}{(l+k-3) !} \leq e^{4 / A_{8}}(l+k-2)
$$

we have

$$
\begin{gathered}
\left(\left|t_{0}\right|+\varepsilon^{4}\right)^{4 / 5} \sum_{\mathbf{k}} \frac{k ! 4^{k_{4}}}{k_{1} ! k_{2} ! k_{3} ! k_{4} !}\left\|t^{(l-3) / 5} \partial_{x}^{l-3}\left(P^{k_{1}} u \quad P^{k_{2}} u P^{k_{3}} u\right)\right\|_{H^{1}\left(I_{x_{0}}\right)} \\
\leq\left(\left|t_{0}\right|+\varepsilon^{4}\right)^{4 / 5} \sum_{1} \sum_{\mathbf{k}} \frac{(l-3) !}{l_{1} ! l_{2} ! l_{3} !} \frac{k ! 4^{k_{4}}}{k_{1} ! k_{2} ! k_{3} ! k_{4} !}\left\|\left(t^{1 / 5} \partial_{x}\right)^{l_{1}} P^{k_{1}} u\right\|_{H^{1}\left(I_{x_{0}}\right)} \\
\quad \times\left\|\left(t^{1 / 5} \partial_{x}\right)^{l_{2}} P^{k_{2}} u\right\|_{H^{1}\left(I_{x_{0}}\right)}\left\|\left(t^{1 / 5} \partial_{x}\right)^{l_{3}} P^{k_{3}} u\right\|_{H^{1}\left(I_{x_{0}}\right)}
\end{gathered}
$$


Advances in Mathematical Physics

$$
\begin{aligned}
\leq & \left(\left|t_{0}\right|+\varepsilon^{4}\right)^{4 / 5} K_{7}^{3}(l+k-3) ! A_{8}^{l+k-3} \\
& \times \sum_{1} \sum_{\mathbf{k}} \frac{\left(4 A_{8}^{-1}\right)^{k_{4}}}{k_{4} !} \frac{\left(l_{1}+k_{1}\right) !}{k_{1} ! l_{1} !} \frac{\left(l_{2}+k_{2}\right) !}{k_{2} ! l_{2} !} \frac{\left(l_{3}+k_{3}\right) !}{k_{3} ! l_{3} !} \frac{(l-3) ! k !}{(l+k-3) !} \\
\leq & \left(\left|t_{0}\right|+\varepsilon^{4}\right)^{4 / 5} K_{7}^{3} A_{8}^{l+k-3} e^{4 / A_{8}}(l+k-2) ! \\
\leq & \frac{1}{5} K_{7} A_{8}^{k+l}(k+l-2) ! .
\end{aligned}
$$

Similarly,

$$
\begin{aligned}
&\left(\left|t_{0}\right|\right.\left.+\varepsilon^{4}\right)^{4 / 5} \sum_{\mathbf{k}} \frac{k ! 3^{k_{4}}}{k_{1} ! k_{2} ! k_{3} ! k_{4} !}\left\|t^{l-3 / 5} \partial_{x}^{l-3}\left(\partial_{x} P^{k_{1}} u \partial_{x} P^{k_{2}} u\right)\right\|_{H^{1}\left(I_{x_{0}}\right)} \\
& \leq\left(\left|t_{0}\right|+\varepsilon^{4}\right)^{4 / 5} \sum_{l-3=l_{1}+l_{2}} \sum_{\mathbf{k}} \frac{(l-3) !}{l_{1} ! l_{2} !} \frac{3^{k_{3}} k !}{k_{1} ! k_{2} ! k_{3} ! k_{4} !} \\
& \quad \times\left\|\left(t^{1 / 5} \partial_{x}\right)^{l_{1}} \partial_{x} P^{k_{1}} u\right\|_{H^{1}\left(I_{x_{0}}\right)}\left\|\left(t^{1 / 5} \partial_{x}\right)^{l_{2}} \partial_{x} P^{k_{2}} u\right\|_{H^{1}\left(I_{x_{0}}\right)} \\
& \leq\left(\left|t_{0}\right|+\varepsilon^{4}\right)^{4 / 5}\left|t_{0}-\varepsilon^{4}\right|^{-2 / 5} \sum_{l-1=m_{1}+m_{2}} \sum_{\mathbf{k}} \frac{(l-3) ! m_{1} m_{2}}{m_{1} ! m_{2} !} \frac{3^{k_{3}} k !}{k_{1} ! k_{2} ! k_{3} ! k_{4} !} \\
& \quad \times\left\|\left(t^{1 / 5} \partial_{x}\right)^{m_{1}} P^{k_{1}} u\right\|_{H^{1}\left(I_{x_{0}}\right)}\left\|\left(t^{1 / 5} \partial_{x}\right)^{m_{2}} P^{k_{2}} u\right\|_{H^{1}\left(I_{x_{0}}\right)^{\prime}}
\end{aligned}
$$

where $m_{1}=l_{1}+1$ and $m_{2}=l_{2}+1$. By $m_{1}^{2} \geq m_{1}$ and $m_{2}^{2} \geq m_{2}$, we have

$$
m_{1} m_{2}=\frac{1}{2}\left\{(l-1)^{2}-\left(m_{1}^{2}+m_{2}^{2}\right)\right\} \leq\left\{(l-1)^{2}-\left(m_{1}+m_{2}\right)\right\} \leq(l-2)(l-1) .
$$

Thus, we have by (4.56), (4.43)

$$
\begin{gathered}
\left(\left|t_{0}\right|+\varepsilon^{4}\right)^{4 / 5}\left(\left|t_{0}\right|+\varepsilon^{4}\right)^{-2 / 5} \sum_{l-1=m_{1}+m_{2}} \sum_{\mathbf{k}} \frac{(l-3) !}{m_{1} ! m_{2} !} \frac{3^{k_{3}} k !}{k_{1} ! k_{2} ! k_{3} ! k_{4} !} \\
\times\left\|\left(t^{1 / 5} \partial_{x}\right)^{m_{1}} P^{k_{1}} u\right\|_{H^{1}\left(I_{x_{0}}\right)}\left\|\left(t^{1 / 5} \partial_{x}\right)^{m_{2}} P^{k_{2}} u\right\|_{H^{1}\left(I_{x_{0}}\right)}
\end{gathered}
$$




$$
\begin{aligned}
\leq & \left(\left|t_{0}\right|+\varepsilon^{4}\right)^{4 / 5}\left|t_{0}-\varepsilon^{4}\right|^{-2 / 5} K_{7}^{2}(l+k-1) ! A_{g}^{l+k-1} \\
& \times \sum_{l-1=m_{1}+m_{2}} \sum_{\mathbf{k}} \frac{\left(3 A_{8}^{-1}\right)^{k_{3}} !}{k_{3} !} \frac{A_{8}^{-k_{4}} !}{k_{4} !} \frac{\left(m_{1}+k_{1}\right) !}{k_{1} m_{1} !} \frac{\left(m_{2}+k_{2}\right) !}{k_{2} m_{2} !} \frac{(l-1) ! k !}{(l+k-1) !} \\
\leq & \left(\left|t_{0}\right|+\varepsilon^{4}\right)^{4 / 5}\left|t_{0}-\varepsilon^{4}\right|^{-2 / 5} K_{7}^{2} e^{4 / A_{s}}(l+k) ! A_{8}^{l+k-1} \\
\leq & \frac{1}{5} K_{7} A_{8}^{k+l+1}(K+l) !
\end{aligned}
$$

Combining (4.48)-(4.57), we have (4.46). This completes the proof.

Proposition 4.9. Suppose that (4.43) holds for all $k, l=0,1,2, \ldots$. Then, there exists $A_{9}>0$ depending on $A_{8},\left(t_{0}, x_{0}\right)$, and $\varepsilon$ such that

$$
\sup _{t \in I_{t_{0}}}\left\|\partial_{t}^{m} \partial_{x}^{l} u\right\|_{H^{1}\left(I_{x_{0}}\right)} \leq K_{7} A_{9}^{m+l}(m+l) !
$$

holds for all $m, l=0,1,2, \ldots$

Proof. By induction on $m$, we prove

$$
\left\|\left(x \partial_{x}\right)^{m} \partial_{x}^{l} P^{k} u\right\|_{H^{1}\left(I_{x_{0}}\right)} \leq K_{7} A_{10}^{k+l+m} B_{1}^{m}(k+l+m) ! \quad k, l, m=0,1,2, \ldots
$$

In the case $m=0$, we have by $(4.43)$

$$
\begin{aligned}
\sup _{t \in I_{t_{0}}}\left\|\partial_{x}^{l} P^{k} u\right\|_{H^{1}\left(I_{x_{0}}\right)} & \leq K_{7} A_{10}^{l} A_{8}^{k}(k+l) ! \\
& \leq K_{7} A_{10}^{k+l}(k+l) !, \quad k, l=0,1,2, \ldots
\end{aligned}
$$

where $A_{10}=\max \left\{A_{8}\left|t_{0}-\varepsilon^{4}\right|^{-1 / 5}, A_{8}\right\}$. We assume (4.59) is valid up to any $m$. Since

$$
\partial_{x}\left(x \partial_{x}\right)^{m}=\left(x \partial_{x}+1\right)^{m} \partial_{x} \quad(m=0,1,2, \ldots),
$$


Advances in Mathematical Physics

we have

$$
\begin{aligned}
\|( & \left(\partial_{x}\right)^{m+1} \partial_{x}^{l} P^{k} u \|_{H^{1}\left(I_{x_{0}}\right)} \\
\leq & \left(\left|x_{0}\right|+\varepsilon^{4}+1\right)\left\|\left(x \partial_{x}+1\right)^{m} \partial_{x}^{l+1} P^{k} u\right\|_{H^{1}\left(I_{x_{0}}\right)} \\
\leq & \left(\left|x_{0}\right|+\varepsilon^{4}+1\right) \sum_{m_{1}=0}^{m} \frac{m !}{m_{1} !\left(m-m_{1}\right) !}\left\|\left(x \partial_{x}\right)^{m_{1}} \partial_{x}^{l+1} P^{k} u\right\|_{H^{1}\left(I_{x_{0}}\right)} \\
\leq & \left(\left|x_{0}\right|+\varepsilon^{4}+1\right) K_{7} \mathrm{~A}_{10}^{k+m+l+1} B_{1}^{m}(k+m+l+1) ! \\
& \times \sum_{m_{1}=0}^{m} \frac{\left(A_{10} B_{1}\right)^{-\left(m-m_{1}\right)}}{\left(m-m_{1}\right) !} \frac{m !}{m_{1} !} \frac{\left(k+m_{1}+l+1\right) !}{(k+m+l+1) !} \\
\leq & \left(\left|x_{0}\right|+\varepsilon^{4}+1\right) e^{-A_{10} B_{1}} K_{7} A_{10}^{k+m+l+1} B_{1}^{m}(k+m+l+1) ! \\
\leq & K_{7} A_{10}^{k+m+l+1} B_{1}^{m+1}(k+m+l+1) !,
\end{aligned}
$$

where $B_{1} \geq\left(\left|x_{0}\right|+\varepsilon^{4}+1\right) e^{-A_{10} B_{1}}$. Since $t \partial_{t}=\left(P-x \partial_{x}\right) / 5$ and

$$
P^{n_{1}} \partial_{x}^{n_{2}}=\partial_{x}^{n_{2}}\left(P-n_{2}\right)^{n_{1}} \quad\left(n_{1}, n_{2}=0,1,2, \ldots\right),
$$

it follows from (4.59) that

$$
\begin{aligned}
\left\|\left(t \partial_{t}\right)^{m} \partial_{x}^{l} u\right\|_{H^{1}\left(I_{x_{0}}\right)} & \leq 5^{-m} \sum_{m=m_{1}+m_{2}} \frac{m}{m_{1} ! m_{2} !}\left\|\left(x \partial_{x}\right)^{m_{1}} P^{m_{2}} \partial_{x}^{l} u\right\|_{H^{1}\left(I_{x_{0}}\right)} \\
& \leq 5^{-m} \sum_{m=m_{1}+m_{2}} \frac{m}{m_{1} ! m_{2} !}\left\|\left(x \partial_{x}\right)^{m_{1}} \partial_{x}^{l}(P-l)^{m_{2}} u\right\|_{H^{1}\left(I_{x_{0}}\right)} \\
& \leq 5^{-m} \sum_{\mathbf{m}} \frac{m !}{m_{1} ! m_{2} ! m_{3} !} l^{m_{3}}\left\|\left(x \partial_{x}\right)^{m_{1}} \partial_{x}^{l} P^{m_{2}} u\right\|_{H^{1}\left(I_{x_{0}}\right)} \\
& \leq 5^{-m} K_{7}(l+m) ! \sum_{\mathbf{m}} \frac{m !}{m_{1} ! m_{2} ! m_{3} !} A_{10}^{l+m-m_{3}} B_{1}^{m_{1}} l^{m_{3}} \frac{\left(m_{1}+m_{2}+l\right) !}{(l+m) !} \\
& \leq 5^{-m} K_{7} A_{10}^{l+m} B_{1}^{m}(l+m) ! \sum_{\mathbf{m}} \frac{m !}{m_{1} ! m_{2} ! m_{3} !}\left(A_{11} B_{1}\right)^{-m_{3}} B_{1}^{-m_{2}} \\
& \leq 5^{-m} K_{7} \max \left\{A_{10} B_{1}, B_{1}\right\}^{l+m}(l+m) !\left(1+\left(A_{10} B_{1}\right)^{-1}+B_{1}^{-1}\right)^{m} \\
& \leq K_{7} A_{11}^{l+m}(l+m) !,
\end{aligned}
$$


where $A_{11}=\max \left\{1,5^{-1}\left(1+\left(A_{10} B_{1}\right)^{-1}+B_{1}^{-1}\right)\right\} \cdot \max \left\{A_{10} B_{1}, B_{1}\right\}$. Thus,

$$
\left\|\left(t \partial_{t}\right)^{m} \partial_{x}^{l} u\right\|_{H^{1}\left(I_{x_{0}}\right)} \leq K_{7} A_{11}^{l+m}(l+m) ! \quad l, m=0,1,2, \ldots
$$

By induction on $j$ we prove that (4.65) implies

$$
\left\|\left(t \partial_{t}\right)^{m} \partial_{t}^{j} \partial_{x}^{l} u\right\|_{H^{1}\left(I_{x_{0}}\right)} \leq K_{7} A_{11}^{j+m+l} B_{2}^{j}(j+m+l) ! \quad j, l, m=0,1,2, \ldots
$$

In the case $j=0$, we have by (4.65)

$$
\sup _{t \in I_{t_{0}}}\left\|\left(t \partial_{t}\right)^{m} \partial_{x}^{l} u\right\|_{H^{1}\left(I_{x_{0}}\right)} \leq K_{7} A_{11}^{l+m}(l+m) ! \quad k, l=0,1,2, \ldots
$$

We assume that (4.66) is valid up to any $j$. Noting that

$$
\left(t \partial_{t}\right)^{m} \partial_{t}=\partial_{t}\left(t \partial_{t}-1\right)^{m} \quad(j=0,1,2, \ldots)
$$

we have

$$
\begin{aligned}
\left\|\left(t \partial_{t}\right)^{m} \partial_{t}^{j+1} \partial_{x}^{l} u\right\|_{H^{1}\left(I_{x_{0}}\right)} \leq & \left\|\partial_{t}\left(t \partial_{t}-1\right)^{m} \partial_{t}^{j} \partial_{x}^{l} u\right\|_{H^{1}\left(I_{x_{0}}\right)} \\
\leq & \left|t_{0}-\varepsilon^{4}\right|^{-1} \sum_{m_{1}=0}^{m} \frac{m !}{m_{1} !\left(m-m_{1}\right) !}\left\|\left(t \partial_{t}\right)^{m_{1}+1} \partial_{t}^{j} \partial_{x}^{l} u\right\|_{H^{1}\left(I_{x_{0}}\right)} \\
\leq & \left|t_{0}-\varepsilon^{4}\right|^{-1} K_{7} A_{11}^{j+l+m+1} B_{2}^{j}(j+l+m+1) ! \\
& \times \sum_{m_{1}=0}^{m} \frac{A_{11}^{-\left(m-m_{1}\right)}}{\left(m-m_{1}\right) !} \frac{m !}{m_{1} !} \frac{\left(j+l+m_{1}+1\right) !}{(j+l+m+1) !} \\
\leq & \left|t_{0}-\varepsilon^{4}\right|^{-1} e^{-A_{11}} K_{7} A_{11}^{j+l+m+1} B_{2}^{j}(j+l+m+1) ! \\
\leq & K_{7} A_{11}^{j+l+m+1} B_{2}^{j+1}(j+l+m+1) !
\end{aligned}
$$

where $B_{2} \geq\left|t_{0}-\varepsilon^{4}\right|^{-1} e^{-A_{11}}$. Thus (4.66) holds.

Choosing $m=0$ and $A_{9}=\max \left\{A_{11} B_{2}, A_{11}\right\}$ in (4.66), we have (4.58). This completes the proof of Proposition 4.9 .

\section{Acknowledgment}

The author is deeply grateful to the referees for having checked the manuscript carefully. 


\section{References}

[1] P. D. Lax, "Periodic solutions of the KdV equation," Communications on Pure and Applied Mathematics, vol. 28, pp. 141-188, 1975.

[2] G. Ponce, "Lax pairs and higher order models for water waves," Journal of Differential Equations, vol. 102, no. 2, pp. 360-381, 1993.

[3] D. J. Benney, "A general theory for interactions between short and long waves," Studies in Applied Mathematics, vol. 56, no. 1, pp. 81-94, 1976/77.

[4] P. J. Olver, "Hamiltonian and non-Hamiltonian models for water waves," in Trends and Applications of Pure Mathematics to Mechanics, vol. 195 of Lecture Notes in Physics, pp. 273-290, Springer, Berlin, Germany, 1984.

[5] To. Kato and K. Masuda, "Nonlinear evolution equations and analyticity-I," Annales de l'Institut Henri Poincaré. Analyse Non Linéaire, vol. 3, no. 6, pp. 455-467, 1986.

[6] N. Hayashi, "Analyticity of solutions of the Korteweg-de Vries equation," SIAM Journal on Mathematical Analysis, vol. 22, no. 6, pp. 1738-1743, 1991.

[7] K. Kato and T. Ogawa, "Analyticity and smoothing effect for the Korteweg de Vries equation with a single point singularity," Mathematische Annalen, vol. 316, no. 3, pp. 577-608, 2000.

[8] N. Hayashi, K. Tomoeda, and T. Yanagisawa, "Analyticity for higher order nonlinear dispersive equations," in Current Advances in Nonlinear Analysis and Related Topics, vol. 32 of GAKUTO International Series. Mathematical Sciences and Applications, pp. 111-130, Gakkōtosho, Tokyo, Japan, 2010.

[9] C. E. Kenig, G. Ponce, and L. Vega, "A bilinear estimate with applications to the KdV equation," Journal of the American Mathematical Society, vol. 9, no. 2, pp. 573-603, 1996.

[10] J. Bourgain, "Fourier transform restriction phenomena for certain lattice subsets and applications to nonlinear evolution equations-I. Schrödinger equations," Geometric and Functional Analysis, vol. 3, no. 2, pp. 107-156, 1993.

[11] J.-C. Saut, "Quelques généralisations de l'équation de Korteweg-de Vries-II," Journal of Differential Equations, vol. 33, no. 3, pp. 320-335, 1979.

[12] M. Schwarz, Jr., "The initial value problem for the sequence of generalized Korteweg-de Vries equations," Advances in Mathematics, vol. 54, no. 1, pp. 22-56, 1984.

[13] C. E. Kenig, G. Ponce, and L. Vega, "On the hierarchy of the generalized KdV equations," in Singular Limits of Dispersive Waves, vol. 320, pp. 347-356, Plenum, New York, NY, USA, 1994.

[14] C. E. Kenig, G. Ponce, and L. Vega, "Higher-order nonlinear dispersive equations," Proceedings of the American Mathematical Society, vol. 122, no. 1, pp. 157-166, 1994.

[15] S. Kwon, "On the fifth-order KdV equation: local well-posedness and lack of uniform continuity of the solution map," Journal of Differential Equations, vol. 245, no. 9, pp. 2627-2659, 2008.

[16] Ta. Kato, "Local well-posedness for the fifth order KdV equation," submitted in Funccialaj Ekvacioj, http://arxiv.org/abs/1011.3956.

[17] K. Tomoeda, "Analyticity and smoothing effect for the fifth order KdV type equation," Proceedings of the Japan Academy, Series A, vol. 86, no. 7, pp. 101-106, 2010.

[18] C. E. Kenig, G. Ponce, and L. Vega, "The Cauchy problem for the Korteweg-de Vries equation in Sobolev spaces of negative indices," Duke Mathematical Journal, vol. 71, no. 1, pp. 1-21, 1993.

[19] K. Tomoeda, "Linear estimates for the Cauchy problem of the fifth order KdV type equation," Annual Reports of Graduate School of Humanities and Sciences Nara Women's University, vol. 25, pp. 295-302, 2009.

[20] J. Ginibre, Y. Tsutsumi, and G. Velo, "On the Cauchy problem for the Zakharov system," Journal of Functional Analysis, vol. 151, no. 2, pp. 384-436, 1997.

[21] W. Chen, J. Li, C. Miao, and J. Wu, "Low regularity solutions of two fifth-order KdV type equations," Journal d'Analyse Mathématique, vol. 107, pp. 221-238, 2009.

[22] D. Bekiranov, T. Ogawa, and G. Ponce, "Weak solvability and well-posedness of a coupled Schrödinger-Korteweg de Vries equation for capillary-gravity wave interactions," Proceedings of the American Mathematical Society, vol. 125, no. 10, pp. 2907-2919, 1997. 


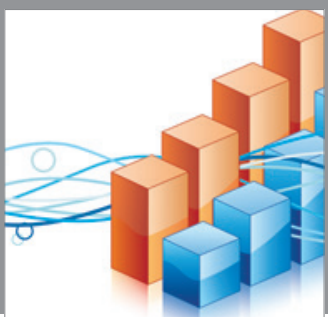

Advances in

Operations Research

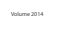

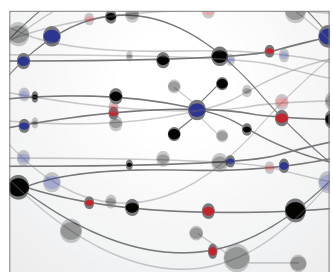

\section{The Scientific} World Journal
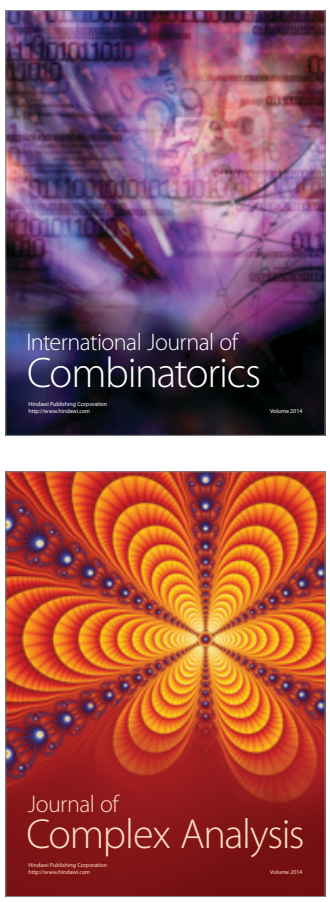

International Journal of

Mathematics and

Mathematical

Sciences
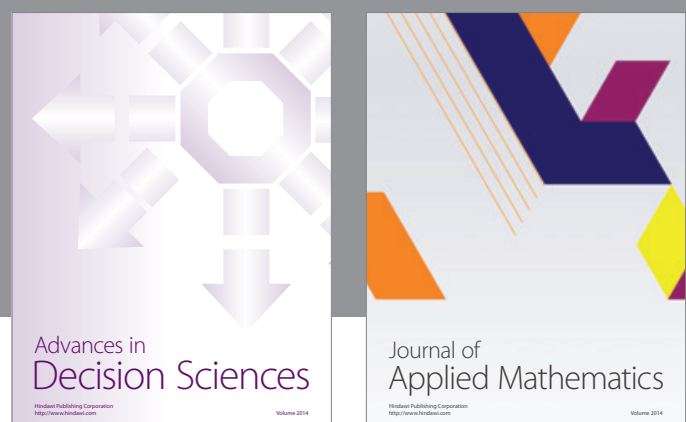

Journal of

Applied Mathematics
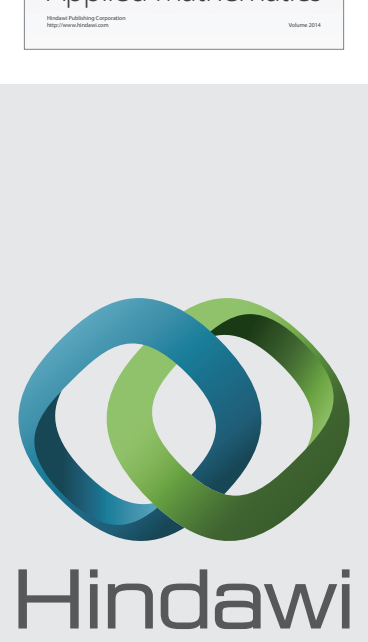

Submit your manuscripts at http://www.hindawi.com
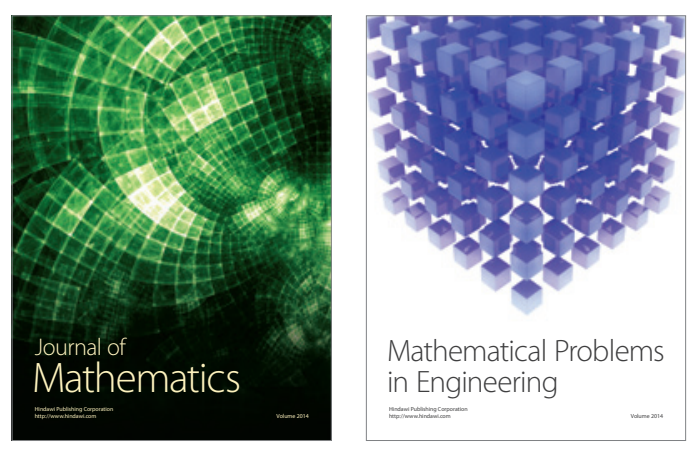

Mathematical Problems in Engineering
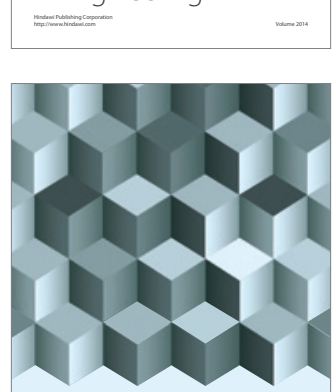

Journal of

Function Spaces
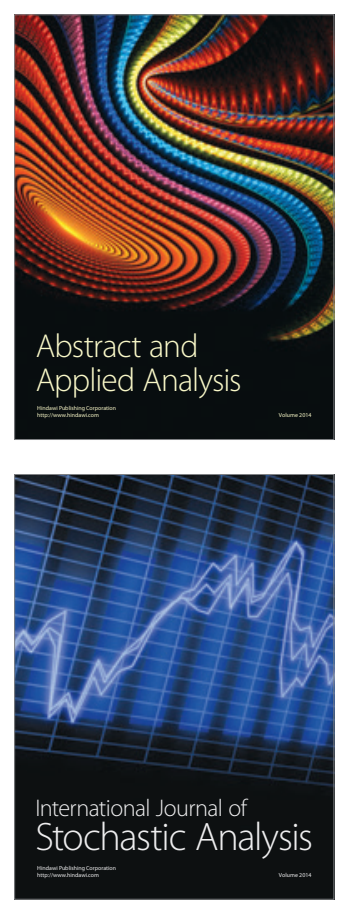

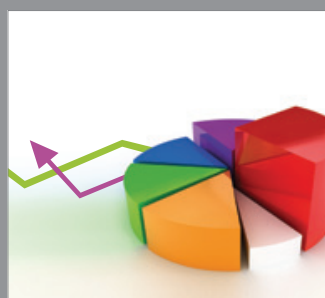

ournal of

Probability and Statistics

Promensencen
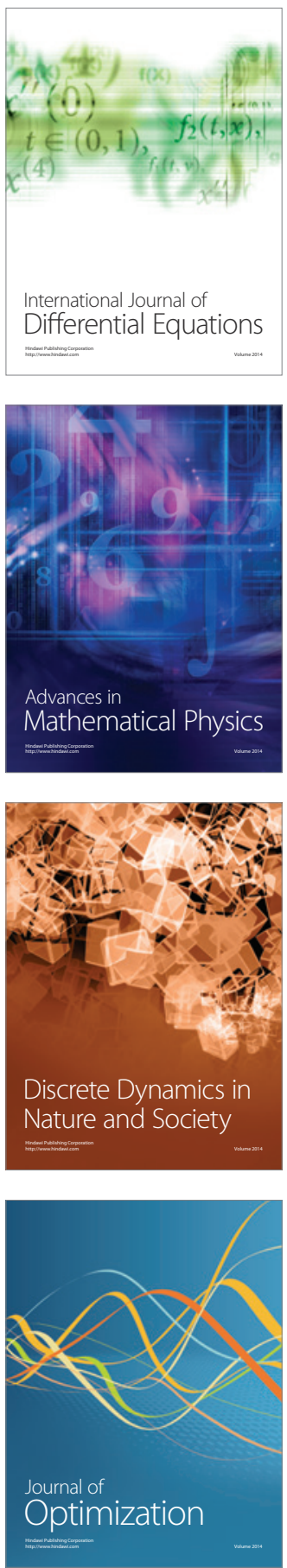